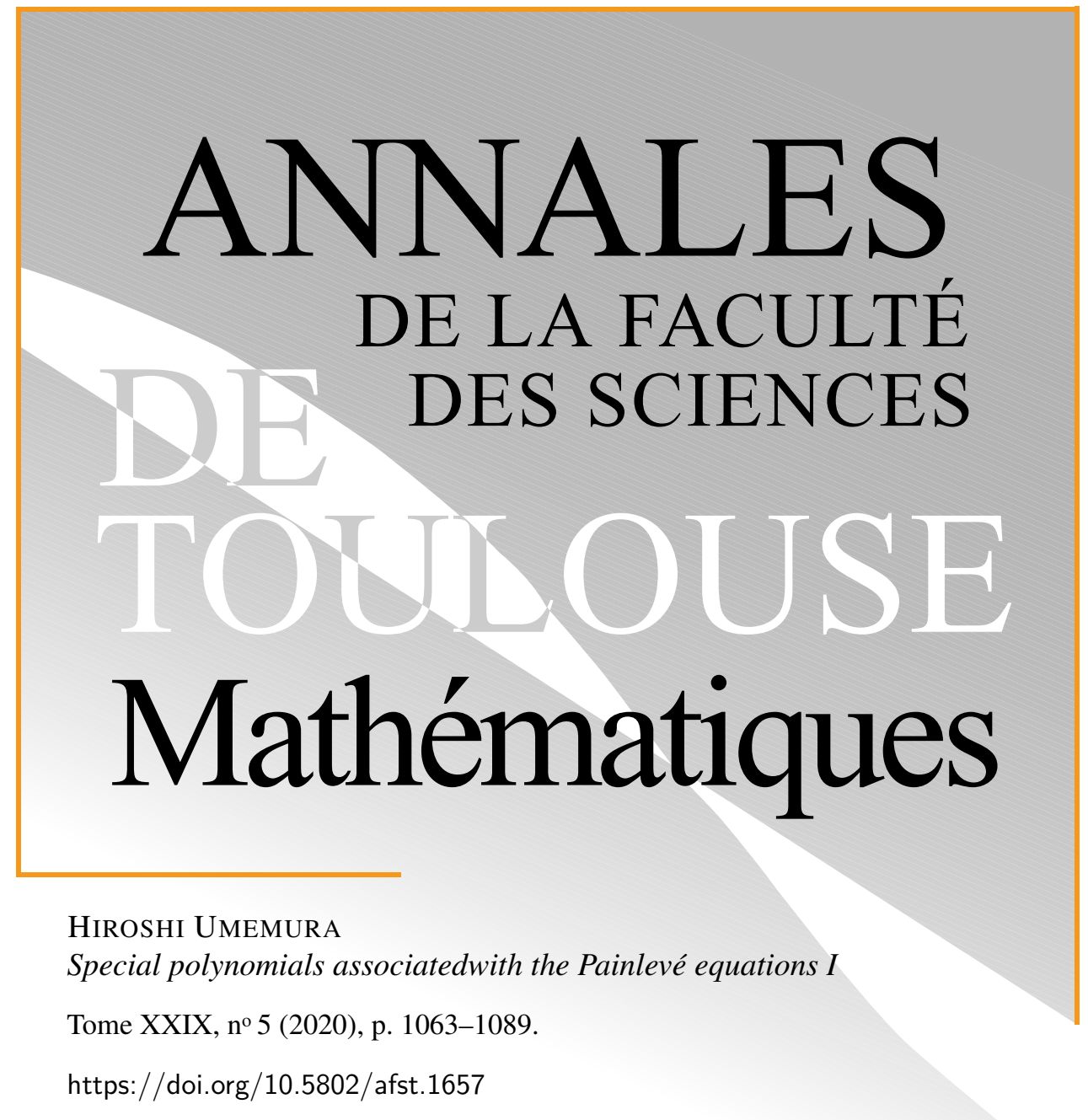

HIROSHI UMEMURA

Special polynomials associatedwith the Painleve equations I

Tome XXIX, nº 5 (2020), p. 1063-1089.

https://doi.org/10.5802/afst.1657

(C) Université Paul Sabatier, Toulouse, 2020.

L'accès aux articles de la revue «Annales de la faculté des sciences de Toulouse Mathématiques » (http://afst.centre-mersenne.org/) implique l'accord avec les conditions générales d'utilisation (http://afst.centre-mersenne.org/legal/). Les articles sont publiés sous la license CC-BY 4.0.

(c) $\underset{B \mathrm{BY}}{(1)}$

Publication membre du centre

Mersenne pour l'édition scientifique ouverte MERSENNE http://www.centre-mersenne.org/ 


\title{
Special polynomials associated with the Painlevé equations I
}

\author{
Hiroshi UMEMURA ${ }^{(1)}$
}

\begin{abstract}
The Painlevé equations have rational or algebraic solutions on special parameters. We can find rational or algebraic solutions of the Painlevé equations as fixed points of the Bäcklund transformations. The $\tau$ function of the rational or algebraic solution can be written as the product of a special polynomial and an exponential factor. Since a series of $\tau$ functions satisfies the Toda equation, we obtain a recursive relation of the special polynomials. The coefficients of the special polynomials for the sixth Painlevé equation are described by the Young diagram.

(The original manuscript by the author was submitted to the proceeding of the Montreal conference in 1996, which were not published. The abstract was not part of the original manuscript and has not been written by the author.)
\end{abstract}

RÉsumé. - Pour certaines valeurs spéciales des paramètres, les équations de Painlevé ont des solutions algébriques ou rationnelles. Elles sont associées aux points fixes des transformations de Bäcklund. La fonction $\tau$ de la solution rationnelle ou algébrique peut alors être écrite comme un produit de polynômes spéciaux et d'un facteur exponentiel. Puisque une série de fonctions $\tau$ satisfait l'équation de Toda, nous obtenons une relation de récurrence pour les polynômes spéciaux. Pour la sixième équation de Painlevé les coefficients des polynômes spéciaux sont décrits à l'aide de diagrammes de Young.

(Le manuscrit original a été soumis aux comptes-rendus de la conférence de Montréal en 1996, qui n'ont pas été publiés. Le résumé ne faisait pas partie du manuscrit original et il n'a pas été rédigé par l'auteur.)

\section{Introduction}

We have systems of classical orthogonal polynomials such as the Jacobi polynomials, the Laguerre polynomials, the Hermite polynomials, etc. They are solutions of linear differential equations of the second order: Namely the hypergeometric differential equations or their confluents.

(1) Graduate School of Polymathematics, Nagoya University 
Example 1.1. - The Hermite polynomials. Let us set

$$
H_{n}(t):=(-1)^{n} e^{t^{2} / 2} \frac{\mathrm{d}^{n} e^{-t^{2} / 2}}{\mathrm{~d} t^{n}}
$$

for every non-negative integer $n$. Then $H_{n}(t)$ is a polynomial of degree $n$, the Hermite polynomial and is a solution of the Hermite equation

$$
y^{\prime \prime}-t y^{\prime}+n y=0 .
$$

The Hermite polynomials are orthogonal each other, i.e.

$$
\int_{-\infty}^{\infty} H_{m}(t) H_{n}(t) e^{-t^{2} / 2} \mathrm{~d} t=\delta_{m n} n ! \sqrt{2 \pi} .
$$

We have a recurrence formula

$$
H_{n+1}(t)=H_{n}(t) t-n H_{n-1}(t) .
$$

As is well-known, we have the following diagram of confluence of the linear differential equations

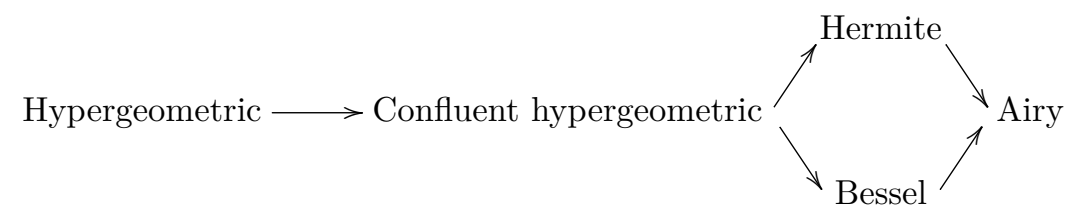

(cf.[12]).

The diagram above is compatible with the degenerations of the Painlevé equations

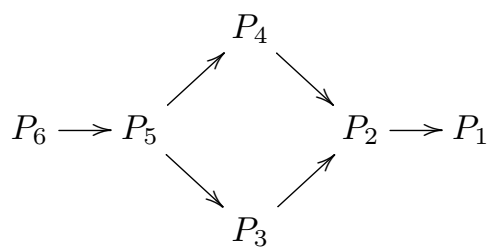

Namely the hypergeometric equation is a part of the sixth Painleve equation, the confluent hypergeometric the fifth and so on. Since the Jacobi polynomials and the Legendre polynomials satisfy hypergeometric equations, they are contained in the sixth Painlevé equation. Similarly the Laguerre polynomials that satisfy a confluent hypergeometric equation, and the Hermite polynomials are respectively included in the fifth and the fourth Painlevé equations.

The Painlevé equations, however, involve also non-classical polynomials. Yablonskii [13] and Vorob'ev [11] discovered such polynomials in $P_{2}$ and the Okamoto in $P_{4}$. So the following natural question arises. 
QUESTION 1.2. - Are there non-classical special polynomials related with the other Painlevé equations?

In fact, we can generate many special polynomials using the Painlevé equations. We present in this note some of them. The other polynomials and the details will appear in [8]. Things go miraculously well. Is it because the Painlevé functions (=the solutions of the Painlevé equations) deserve special functions? The most interesting series of polynomials arises with algebraic solutions of degree 2 of the sixth equation $P_{6}$, which seem new (cf. Section 6 ). In Section 7 we observe that our polynomials are related with the Young diagrams.

\section{Yablonskii-Vorob'ev polynomials (review)}

The second Painlevé equation

$$
P_{2}(\alpha) \quad y^{\prime \prime}=2 y^{3}+t y+\alpha
$$

is equivalent to the system

$$
S_{2}(b) \quad\left\{\begin{array}{l}
q^{\prime}=p-q^{2}-\frac{t}{2}, \\
p^{\prime}=2 p q+b,
\end{array}\right.
$$

where $\alpha$ and $b$ are related by $b=\alpha+\frac{1}{2}$ (cf. [3]). The system $S_{2}(b)$ is a Hamiltonian system parameterized by $b \in \mathbb{C}$. Namely let $H=\frac{1}{2} p^{2}-\left(q^{2}+\frac{1}{2} t\right) p-b$. Then $S_{2}(b)$ is written as

$$
\left\{\begin{array}{l}
\mathrm{d} q / \mathrm{d} t=\partial H / \partial p \\
\mathrm{~d} p / \mathrm{d} t=-\partial H / \partial q .
\end{array}\right.
$$

We denote by $\operatorname{Sol}_{2}(b)$ the set of solutions of $S_{2}(b)$. We have a transformation

$$
T(b, b-1): \operatorname{Sol}_{2}(b) \rightarrow \operatorname{Sol}_{2}(b-1)
$$

defined by

$$
T(b, b-1)(q, p)=\left(-q+(b-1) /\left(p-2 q^{2}-t\right),-p+2 q^{2}+t\right)
$$

and a transformation

$$
I(b,-b): \operatorname{Sol}_{2}(b) \rightarrow \operatorname{Sol}_{2}(-b)
$$

given by $I(b,-b)(q, p)=(q+b / p, p)$ for $(q, p) \in S_{0} l_{2}(b)$. 


\section{Hiroshi Umemura}

\section{THEOREM 2.1.}

(1) $P_{2}(\alpha)$ has a 1-parameter family of classical solutions for $\alpha \in \frac{1}{2}+\mathbb{Z}$.

(2) $P_{2}(\alpha)$ has a unique rational solution for $\alpha \in \mathbb{Z}$.

(3) Except for those solutions in (1) and (2), other solutions are not classical.

This theorem is the irreducibility of the second Painlevé equation. For the definition of classical functions, see [8, 9] and for a proof of the theorem see [10]. Let us illustrate the theorem by examples.

Example 2.2.

(1). - If $\alpha=-\frac{1}{2}$ or equivalently $b=0$, then $S_{2}(0)$ reduces to

$$
S_{2}(0)\left\{\begin{array}{l}
q^{\prime}=p-q^{2}-\frac{t}{2}, \\
p^{\prime}=2 p q .
\end{array}\right.
$$

So if $q$ is a solution of a Riccati equation $q^{\prime}=-q^{2}-t / 2$, then $(q, 0)$ is a solution of $S_{2}(0)$. The solutions of this type form a 1-parameter family of classical solutions of $S_{2}(0)$. We notice that by setting $y^{\prime} / y=q$, we linearize the Riccati equation to get the Airy equation

$$
y^{\prime \prime}+\frac{1}{2} t y=0
$$

(2). - If $\alpha=0$ so that $b=\frac{1}{2}$, we have

$$
S_{2}\left(\frac{1}{2}\right) \quad\left\{\begin{array}{l}
q^{\prime}=p-q^{2}-\frac{t}{2}, \\
p^{\prime}=2 p q+\frac{1}{2} .
\end{array}\right.
$$

Hence $(q, p)=(0, t / 2)$ is the unique rational solution of $S\left(\frac{1}{2}\right)$. It is natural to ask how we can calculate the unique rational solution of $P_{2}(n)$ for $n \in \mathbb{Z}$. The first idea that comes to our mind is to start from the rational solution $(0, t / 2)$ of $S\left(\frac{1}{2}\right)$ and use the transformations $T(b, b-1)$ and $I(b,-b)$. There is a very elegant method due to Yabloskii and Vorob'ev.

Let us define rational functions $T_{m}(t)$ for $m \in \mathbb{N}$ recursively by

$$
T_{m}(t)=\frac{t T_{m-1}^{2}(t)-4\left(T_{m-1}^{\prime \prime}(t) T_{m-1}(t)-T_{m-1}^{\prime 2}(t)\right)}{T_{m-2}(t)},
$$

with $T_{0}(t)=T_{1}(t)=1$. We can show that for every $m \in \mathbb{N}, T_{m}(t)$ is a monic polynomial with integral coefficients of degree $m(m-1) / 2$, which we call the Yablonskii-Vorob'ev polynomials (see 8.1, [11] and [13]). We can show that $q_{m}=T_{m+1}^{\prime} / T_{m+1}-T_{m}^{\prime} / T_{m}$ is the unique rational slution of $P_{2}(-m-1)$ for 
$m \in \mathbb{N}$. We want to generalize the Yablonskii-Vorob'ev polynomials. To this end, we must clarify the following points.

(1) How to find rational or more generally algebraic solutions of the Painlevé equations?

(2) Where does the recurrence formula (2.2) come from?

(3) How to prove that the $T_{m}(t)$ 's are in fact polynomials?

\section{How to find rational or algebraic solutions}

To this end let us review how the transformations $T(b, b-1), I(b,-b)$ are introduced ([3]). As we explained above $S_{2}(b)$ is the Hamiltonian system (2.1) with the Hamiltonian $H(b, t, q, p)=\frac{1}{2} p^{2}-\left(q^{2}+\frac{1}{2} t\right)-b q$. Let us set $h(b, q(t), p(t))=H(b, t, q(t), p(t))$ for a solution $(q(t), p(t))$ of $S_{2}(b)$. Then $h(b, q, p)$, which is a function of $t$, satisfies

$$
E_{2}(b) \quad\left(\frac{\mathrm{d}^{2} h}{\mathrm{~d} t^{2}}\right)^{2}+4\left(\frac{\mathrm{d} h}{\mathrm{~d} t}\right)^{3}+2 \frac{\mathrm{d} h}{\mathrm{~d} t}\left(t \frac{\mathrm{d} h}{\mathrm{~d} t}-h\right)-\frac{1}{4} b^{2}=0 .
$$

The following lemmas are due to Okamoto [3].

Lemma 3.1. - Let $(q, p)$ be a generic solution of $S_{2}(b)$. Namely we have

$$
\text { tr.d. }[\mathbb{Q}(b, t)(q, p): \mathbb{Q}(b, t)]=2 .
$$

Then $h(b, q, p)$ is a generic solution of $E(b)$, i.e. we have

$$
\operatorname{tr.d.}[\mathbb{Q}(b, t)\langle h(b, q, p)\rangle: \mathbb{Q}(b, t)]=2 .
$$

Here we denote by $\mathbb{Q}(b, t)\langle h(b, q, p)\rangle$ the differential field generated by $h(b, q, p)$ over $\mathbb{Q}(b, t)$.

Lemma 3.2. - If $(q(t), p(t))$ be a generic solution of $S_{2}(b)$, then $\mathbb{Q}(b, t)(q(t), p(t))$ coincides with $\mathbb{Q}(b, t)\langle h(t, q(t), p(t)\rangle$. In fact we have

$$
\left\{\begin{array}{l}
q(t)=\frac{2 \frac{\mathrm{d}^{2} h(b, q(t), p(t))}{\mathrm{d} t^{2}}+b}{4 \frac{\mathrm{d} h(b, q(t), p(t))}{\mathrm{d} t}}, \\
p(t)=-2 \frac{\mathrm{d} h(b, q(t), p(t))}{\mathrm{d} t}
\end{array}\right.
$$

(cf. [3, Formula (1.1), p. 227]).

Let now $h$ be a generic solution of $E(b)$. Then it follows from Lemma 3.1 that $(q, p)$ being a generic solution of $S_{2}(b)$, we have a differential $\mathbb{Q}(b, t)$ isomorphism $\mathbb{Q}(b, t)\langle h\rangle \rightarrow \mathbb{Q}(b, t)\langle h(b, q, p)\rangle, h \mapsto h(b, q, p)$. So if we set

$$
\left\{\begin{array}{l}
q(b, h)=\left(2 \mathrm{~d}^{2} h / \mathrm{d} t^{2}+b\right) /(4 \mathrm{~d} h / \mathrm{d} t), \\
p(b, h)=-2 \mathrm{~d} h / \mathrm{d} t,
\end{array}\right.
$$


then $(q(h), p(h))$ is a generic solution of $S_{2}(b)$ and we have

$$
h=h(b, q(b, h), p(b, h))
$$

so that

$$
\mathbb{Q}(b, t)\langle h\rangle=\mathbb{Q}(b, t)(q(b, h), p(b, h)) .
$$

Let $(q, p)$ be a generic solution of $S_{2}(b)$. Then by Lemma $3.1 h(b, q, p)$ is a generic solution of $E(b)$ and hence is a generic solution of $E(-b)$. Therefore we have by Lemma 3.2 and (3.4).

$$
\mathbb{Q}(b, t)(q, p)=\mathbb{Q}(b, t)\langle h(b, q, p)\rangle=\mathbb{Q}(b, t)(q(-b, h), p(-b, h)),
$$

where $h$ on the right should be read $h(b, q, p))$. A calculation using (3.1) shows

$$
\left\{\begin{array}{l}
q(-b, h(b, q, p))=q+b / p \\
p(-b, h(b, q, p))=p
\end{array}\right.
$$

This is the transformation $I(b,-b)$.

Remark 3.3. - The transformation $I(b,-b)(q, p)$ is defined for a generic solution $(q, p)$ of $S_{2}(b)$ but we can show $I(b,-b)(q, p)$ is well-defined for every solution $(q, p)$ of $S_{2}(b)$.

When $b=-b$ or $b=0, I(0,0)$ is an automorphism of $\mathbb{Q}(t)(q, p)$. It follows from $(3.5)$ that $I(0,0)$ is the identity automorphism.

Lemma 3.4. - I $(0,0)$ is identity automorphsim of $\mathbb{Q}(t)(q, p)$.

Now we analyze the transformation $T(b, b-1): \operatorname{Sol}_{2}(b) \rightarrow \operatorname{Sol}_{2}(b-1)$.

Proposition 3.5 (Okamoto). - If $(q, p)$ is a generic solution of $S_{2}$, then $h_{1}(b, q, p):=h(b, q, p)+q$ is a generic solution of $E(b-1)$. The differential subfield

$$
\mathbb{Q}(b, t)\left\langle h_{1}(b, q, p)\right\rangle
$$

coincides with $\mathbb{Q}(b, t)(q, p)$. Namely we have

$$
\mathbb{Q}(b, t)\left\langle h_{1}(b, q, p)\right\rangle=\mathbb{Q}(b, t)(q, p) .
$$

In fact, we have

$$
\left\{\begin{array}{l}
q=\frac{2 \frac{\mathrm{d}^{2} h_{1}(b, q, p)}{\mathrm{d} t^{2}}-b+1}{4 \frac{\mathrm{d} h_{1}(b, q, p)}{\mathrm{d} t}}, \\
p=2 \frac{\mathrm{d} h_{1}(b, q, p)}{\mathrm{d} t}+2 q^{2}+t
\end{array}\right.
$$

(cf. [3, Formula (2.5), p. 235]). Now it follows from Proposition 3.5 and (3.4)

$$
\mathbb{Q}(b, t)(q, p)=\mathbb{Q}(b, t)\left\langle h_{1}(b, q, p)\right\rangle=\mathbb{Q}(b, t)\left(q\left(b-1, h_{1}\right), p\left(b-1, h_{1}\right)\right),
$$


where $h_{1}$ should be read $h_{1}(b, q, p)$. A calculation using (3.2), (3.8) shows

$$
\left\{\begin{array}{l}
q\left(b-1, h_{1}\right)=-q+\frac{b-1}{p-2 q^{2}-t} \\
p\left(b-1, h_{1}\right)=-p+2 q^{2}+t .
\end{array}\right.
$$

This is the transformation $T(b, b-1): \operatorname{Sol}_{2}(b) \rightarrow \operatorname{Sol}_{2}(b-1)$. The value $T(b, b-1)(q, p)$ is defined for a generic solution $(q, p)$ of $S_{2}(b)$ but we can show that it is well-defined for every solution $(q, p)$ of $S_{2}(b)$. Let us now consider the composite of the translation $b \mapsto b-1$ and the reflection at 0 of $\mathbb{C}: b \mapsto b-1 \mapsto-(b-1)=1-b$. So $\frac{1}{2}$ is its fixed point. This affine transformation $b \mapsto 1-b$ of $\mathbb{C}$ lifts to the transformation

$$
I(b-1,1-b) \circ T(b, b-1): \mathbb{Q}(b, t)(q(t), p(t)) \rightarrow \mathbb{Q}(t, b)(q(1-b), p(1-b)) .
$$

It follows from the definition of the transformations that $I(b-1,1-b) \circ$ $T(b, b-1)$ comes from

$\mathbb{Q}(b, t)(q(b), p(b))=\mathbb{Q}(b, t)\left\langle h_{1}(b, q, p)\right\rangle=\mathbb{Q}(b, t)\left(q\left(1-b, h_{1}\right), p\left(1-b, h_{1}\right)\right)$.

At the fixed point $b=\frac{1}{2}$, we get an automorphism $I(b-1,1-b) \circ$ $T(b, b-1)=I\left(-\frac{1}{2}, \frac{1}{2}\right) \circ T\left(\frac{1}{2},-\frac{1}{2}\right): \operatorname{Sol}_{2}\left(\frac{1}{2}\right) \rightarrow \operatorname{Sol}_{2}\left(\frac{1}{2}\right)$. Let us look for a solution

fixed by this automorphism.

$$
\left(q\left(\frac{1}{2}, t\right), p\left(\frac{1}{2}, t\right)\right)=\left(q\left(\frac{1}{2}\right), p\left(\frac{1}{2}\right)\right)
$$

Lemma 3.6. - The following conditions for a solution $\left(q\left(\frac{1}{2}, t\right)\right.$, $\left.p\left(\frac{1}{2}, t\right)\right)$ of $S_{2}\left(\frac{1}{2}\right)$ are equivalent.

(1) $I\left(-\frac{1}{2}, \frac{1}{2}\right) \circ T\left(\frac{1}{2},-\frac{1}{2}\right)\left(q\left(\frac{1}{2}, t\right), p\left(\frac{1}{2}, t\right)\right)=\left(q\left(\frac{1}{2}, t\right), p\left(\frac{1}{2}, t\right)\right)$.

(2) $h_{1}\left(\frac{1}{2}, q, p\right)=h\left(\frac{1}{2}, q, p\right)$.

(3) $q\left(\frac{1}{2}, t\right)=0$.

(4) $\left(q\left(\frac{1}{2}, t\right), p\left(\frac{1}{2}, t\right)\right)=(0, t / 2)$.

Proof. - It follows form (3.2) that we have

$$
\mathbb{Q}(b, t)\langle h(b, q, p)\rangle=\mathbb{Q}(b, t)(q, p)
$$

if $\mathrm{d} h / \mathrm{d} t \neq 0$. We notice $\mathrm{d} h / \mathrm{d} t=0$ implies $b=0$ by $E(b)$. So if $b \neq 0$, we have (3.10). Similarly, it follows form (3.8) that if $b \neq 1$, we have

$$
\mathbb{Q}(b, t)\left\langle h_{1}(b, q, p)\right\rangle=\mathbb{Q}(b, t)(q, p) .
$$

Now since $b=\frac{1}{2}$, we have both (3.11) and (3.12). So $I(b-1,1-b) \circ$ $T(b, b-1)(q, p)$ is defined for every $(q, p) \in \operatorname{Sol}_{2}\left(\frac{1}{2}\right)$ by the equalities (3.11) and $(3.12)$ as for a generic solution: We have $\mathbb{Q}(t)(q, p)=\mathbb{Q}(t)\left\langle h_{1}\left(\frac{1}{2}, q, p\right)\right\rangle=$ $\mathbb{Q}(t)\left(q\left(\frac{1}{2}, h_{1}\right), p\left(\frac{1}{2}, h_{1}\right)\right)$ and $I\left(-\frac{1}{2}, \frac{1}{2}\right) \circ T\left(\frac{1}{2},-\frac{1}{2}\right)(q, p)=\left(q\left(\frac{1}{2}, h_{1}\right), p\left(\frac{1}{2}, h_{1}\right)\right)$. So since $h_{1}\left(\frac{1}{2}, q, p\right)=h\left(\frac{1}{2}, q\left(\frac{1}{2}, h_{1}\right), p\left(\frac{1}{2}, h_{1}\right)\right)$, if $q\left(\frac{1}{2}, h_{1}\right)=q\left(\frac{1}{2}, t\right)$ and $p\left(\frac{1}{2}, h_{1}\right)=p\left(\frac{1}{2}, t\right)$, then we have $h_{1}\left(\frac{1}{2}, q\left(\frac{1}{2}\right), p\left(\frac{1}{2}\right)\right)=h\left(\frac{1}{2}, q\left(\frac{1}{2}\right), p\left(\frac{1}{2}\right)\right)$. This 
shows the conditions (1) and (2) are equivalent. It follows from the definition that the second condition is equivalent to the third. By the first equation of $S_{2}\left(\frac{1}{2}\right), q\left(\frac{1}{2}\right)=0$ if and only if $p\left(\frac{1}{2}\right)=t / 2$.

\section{Remarks 3.7.}

(1). - We have seen that the rational solution $(q, p)=(0, t / 2)$ of $S_{2}\left(\frac{1}{2}\right)$ is the unique fixed solution of the automorphism

$$
I\left(-\frac{1}{2}, \frac{1}{2}\right) \circ T\left(\frac{1}{2},-\frac{1}{2}\right): \mathbb{Q}(t)\left(q\left(\frac{1}{2}\right), p\left(\frac{1}{2}\right)\right) \rightarrow \mathbb{Q}(t)\left(q\left(\frac{1}{2}\right), p\left(\frac{1}{2}\right)\right) .
$$

As Theorem 2.1 shows, $(q, p)=(0, t / 2)$ is the unique rational solution and hence $(0, t / 2)$ is fixed by the birational automorphism $I\left(-\frac{1}{2}, \frac{1}{2}\right) \circ T\left(\frac{1}{2},-\frac{1}{2}\right)$.

(2). - Now we fix the parameter $b \in \mathbb{C}$. Let

$$
\mathcal{X}=\frac{\partial}{\partial t}+\frac{\partial H}{\partial p} \frac{\partial}{\partial q}-\frac{\partial H}{\partial q} \frac{\partial}{\partial p}
$$

which is a vector field on $X=\mathbb{C}^{3}$ with coordinate $(t, q, p)$. We denote by $p: X=\mathbb{C}^{3} \rightarrow \mathbb{C}$ the first projection. A section $(t, q(t), p(t))$ of the projection $p$ is tangent to the vector field $\mathcal{X}$ if and only if $(q(t), p(t))$ satisfies $\mathrm{d} q(t) / \mathrm{d} t=\partial H / \partial p, \mathrm{~d} p(t) / \mathrm{d} t=-\partial H / \partial q$, i.e. $(q(t), p(t))$ is a solution of $S_{2}(b)$. We consider the pair $(X, \mathcal{X})$ and a birational automorphism $f:(X, \mathcal{X}) \rightarrow(X, \mathcal{X})$ that commutes with the vector field $\mathcal{X}$ and with the projection $p$. Let $S \subset X$ be the set of fixed points of the automorphism $f$. Then we can show that if a solution of $S_{2}(b)$ has initial conditions in $S$, then it remains in $S$ or equivalently $S$ is $\mathcal{X}$-invariant. So if the restriction $p \mid s: S \rightarrow \mathbb{C}$ is finite algebraic over a Zariski open set of $\mathbb{C}$, then $S$ gives an algebraic solution of $S_{2}(b)$. In our example, $f=I\left(-\frac{1}{2}, \frac{1}{2}\right) \circ T\left(\frac{1}{2},-\frac{1}{2}\right)$ and $S=\left\{(t, 0, t / 2) \in X=\mathbb{C}^{3} \mid t \in \mathbb{C}\right\}$ so that $p \mid s: S \rightarrow \mathbb{C}$ is biregular and we find the rational solution $(q(t), p(t))=(0, t / 2)$.

Geometrically, regarding now $b \in \mathbb{C}$ as a parameter, we consider the affine space $Z=\mathbb{C}^{4}$ of dimension 4 with coordinate $(b, t ; q, p)$ so that $\mathcal{X}$ is a vector field on $Z$. The transformation $T(b, b-1)$ induces a birational automorphism $T(-1):(Z, \mathcal{X}) \rightarrow(Z, \mathcal{X}),(b, t ; q, p) \mapsto(b-1, t ; T(b, b-1)(q, p))$ lifting the translation $t(-1): \mathbb{C} \rightarrow \mathbb{C}, b \mapsto b-1$ of the parameter space so that the diagram

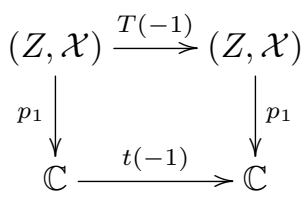

is commutative, where the vertical arrow $p_{1}$ is the first projection. Similarly the transformation $I(b,-b)$ defines a birational automorphism 
$I:(Z, \mathcal{X}) \rightarrow(Z, \mathcal{X}),(b, t ; q, p) \mapsto(-b, t ; I(b,-b)(q, p))$ that lifts the reflection $i: \mathbb{C} \rightarrow \mathbb{C}, b \mapsto-b$ at the origine so that the diagram

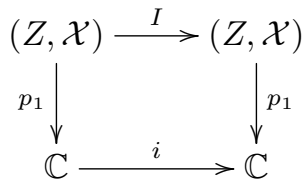

is commutative. Let $G$ be the subgroup of affine transformations of $\mathbb{C}$ generated by the translation $t(-1)$ and the reflection $i$ at the origine of $\mathbb{C}$. So the subgroup $\langle t(-1)\rangle \simeq \mathbb{Z}$ generated by $t(-1)$ is the group of translations by integers and $G=\langle t(-1), i\rangle$ is the semi-direct product $\langle t(-1)\rangle \rtimes\langle i\rangle \simeq \mathbb{Z} \rtimes \mathfrak{S}_{2}$. Let $\widetilde{G}$ be the subgroup of $\mathcal{X}$-birational automorphism of $Z$ generated by $T(-1)$ and $I$. Hence we have a canonical morphism $f: \widetilde{G} \rightarrow G$ such that $f(T(-1))=t(-1)$ and $f(I)=i$. Namely the projection $p_{1}: Z \rightarrow \mathbb{C}$ is equivariant: For every $g \in \widetilde{G}$, we have $p_{1}(g \cdot(b, t ; q, p))=f(g) \cdot\left(p_{1}(b, t ; q, p)\right)$ for all $(b, t ; q, p) \in \mathbb{C}^{4}$. Here the dots mean the operations of $\widetilde{G}$ and $G$ respectively on $Z$ and $\mathbb{C}$. It follows from the definition $I^{2}=\mathrm{Id}$. A calculation shows $(I \circ T(-1))^{2}=\mathrm{Id}$ and hence $\widetilde{G}=\langle T(-1)\rangle \rtimes\langle I\rangle$. Therefore the canonical morphism $f: \widetilde{G}=\langle T(-1)\rangle \rtimes\langle I\rangle \rightarrow G=\langle t(-1)\rangle \rtimes\langle i\rangle$ is an isomorphism. In other words, $G$ operates birationally on $(Z, \mathcal{X})$.

Now we introduce a new parameter space $V:=\left\{\left(v_{1}, v_{2}\right) \in \mathbb{C}^{2} \mid v_{1}+v_{2}=0\right\}$ so that $\{(1,-1),(-1,1)\}$ is the root system of type $A_{1}$. The root lattice is a subgroup $\mathbb{Z}(1,-1)$ of $V$ generated by the roots $(1,-1),(-1,1)$. The affine Weyl group is the group of affine transformations of the affine line $V$ generated by the reflections with respect to the points $(n,-n) \in V, n \in \mathbb{Z}$. We know that the affine Weyl group of type $A_{1}$ is the subgroup of affine transformations of $V$ generated by (i) the translations by the elements of the root lattice $\mathbb{Z}(1,-1)$ and (ii) the reflection at the origin $(0,0)$. The weight lattice is the subgroup of $V$ generated by $\frac{1}{2}(1,-1), \frac{1}{2}(-1,1)$. The extended affine Weyl group is the subgroup of affine transformations of $V$ generated by (i) the translation by the weight lattice and the reflection at the origin $(0,0)$. As Okamoto did it, customarily we identify the parameter space $\mathbb{C}$ of the Painleve equation $P_{2}(\alpha)$ or of the system $S_{2}(b)$ with $V$ by setting $v_{1}=-v_{2}=b=\alpha+\frac{1}{2}$ (cf. [3, 4, 5, 6, 7, 10]). So the subgroup $G$ of the affine transformations of the parameter space $\mathbb{C}$ of the system $S_{2}(b)$ generated by the translation $b \mapsto b+1$ and the reflection at the origin is identified with the affine Weyl group of type $A_{1}$. We had better, however, identify the subgroup $G$ of affine transformations with the extended affine Weyl group. Namely in this paper we set $2 v_{1}=-2 v_{2}=b=\alpha+\frac{1}{2}$.

In real picture, $\left\{(v,-v) \in \mathbb{R}^{2} \mid 0 \leqslant v \leqslant \frac{1}{2}\right\}$ is an alcove or a fundamental domain of the operation of the affine Weyl group on $V_{\mathbb{R}}=\left\{\left(v_{1}, v_{2}\right) \in \mathbb{R}^{2} \mid\right.$ 
$\left.v_{1}+v_{2}=0\right\}$. So with the above identification $\{b \in \mathbb{R} \mid 0 \leqslant b \leqslant 1\}$ is an alcove. Hence $\{b \mid b \in \mathbb{Z}\}$ is the set of walls of the affine Weyl group. So the affine Weyl group is generated by the reflections at $n \in \mathbb{Z}$ of $\mathbb{R}$. Similarly $\left\{b \in \mathbb{R} \mid 0 \leqslant b \leqslant \frac{1}{2}\right\}$ is a fundamental domain of the operation of the extended affine Weyl group on $\mathbb{R}=\{b \in \mathbb{R}\}$. Thus the extended affine Weyl group is generated by the reflections at the point of $\frac{1}{2}+\mathbb{Z}$. Now we can rephrase theorem as follows.

THEOREM 3.8.

(1) $S_{2}(b)$ has a 1-parameter family of classical solutions if $b \in \mathbb{C}$ is a wall of the affine Weyl group of type $A_{1}$.

(2) $S_{2}(b)$ has the unique rational solution if $b \in \mathbb{C}$ is a wall of the extended affine Weyl group but $b$ is not a wall of the affine Weyl group.

(3) Except for those solutions in (1) and (2), no solutions of $S_{2}(b)$ are classical.

The above observation is summarized as follows. The point $b=\frac{1}{2}$ of the parameter space $\mathbb{C}$ is the fixed point of the operation of $g=i \circ t(-1) \in G$. So $g$ induces an automorphism of the fiber $p_{1}^{-1}\left(\frac{1}{2}\right)$ of the first projection $p_{1}: Z \rightarrow \mathbb{C}$ and consequently the automorphism $I\left(-\frac{1}{2}, \frac{1}{2}\right) \circ T\left(\frac{1}{2},-\frac{1}{2}\right)$ : $\mathrm{Sol}_{2}\left(\frac{1}{2}\right) \rightarrow \operatorname{Sol}_{2}\left(\frac{1}{2}\right)$. The rational solution $(q, p)=(0, t / 2)$ is the solution of $S_{2}\left(\frac{1}{2}\right)$ invariant by the automorphism $I\left(-\frac{1}{2}, \frac{1}{2}\right) \circ T\left(\frac{1}{2},-\frac{1}{2}\right)$. So the principle is that first we look for an element $g$ of the extended affine Weyl group that has a fixed point $b=\frac{1}{2}$ on the parameter space $\mathbb{C}$ so that $g$ induces an automorphism of $S_{2}\left(\frac{1}{2}\right)$. Then we look for a solution of $S_{2}\left(\frac{1}{2}\right)$ fixed by the operation of $g$.

\section{Recurrence formula}

We need theory of $\tau$-function. Let us introduce a $\tau$ function of a solution $(q(t), p(t))$ of $S_{2}(b)$ by

$$
\tau^{\prime}(t) / \tau(t)=H(b, q(t), p(t))
$$

where $H=\frac{1}{2} p^{2}-\left(q^{2}+\frac{1}{2} t\right) p-b$ is the Hamiltonian. Let us now fix the parameter $b$ and the solution $(q(b, t), p(b, t))$ of $S_{2}(b)$. We set $\tau_{0}=\tau_{0}(t)$ a $\tau$-function of $q(b, t), p(b, t)$. By iteration of the operations $T(b, b-1)$, $T(b-1, b-2), \ldots$, we get from the given solution $(q(b), p(b))=(q(b, t), p(b, t))$ of $S_{2}(b)$ a solution $(q(b-m), p(b-m))$ of $S_{2}(b-m)$. Let $\tau_{m}=\tau_{m}(t)$ be a $\tau$-function of $(q(b-m), p(b-m))$ so that

$$
\tau_{m}^{\prime} / \tau_{m}=H(b-m, q(b-m), p(b-m)),
$$


Theorem 4.1 (Okamoto [3]). - The $\tau_{m}$ 's satisfy the Toda equation

$$
\mathrm{d}^{2} \log \tau_{m} / \mathrm{d} t^{2}=c(m) \tau_{m-1} \tau_{m+1} / \tau_{m-1}^{2},
$$

where $c(m)$ is a non-zero constant.

Example 4.2. - We have a rational $\operatorname{solution}\left(q\left(\frac{1}{2}\right), p\left(\frac{1}{2}\right)\right)=(0, t / 2)$ of $S_{2}\left(\frac{1}{2}\right)$ so that $H\left(\frac{1}{2}, t, q\left(\frac{1}{2}\right), p\left(\frac{1}{2}\right)\right)=-\frac{1}{8} t^{2}$. So since $\tau_{0}$ is a solution of $\tau_{0}^{\prime}=$ $\left(-t^{2} / 8\right) \tau_{0}, \tau_{0}=\exp \left(-\int t^{2} / 8 \mathrm{~d} t\right)=\exp \left(-t^{3} / 24\right)$. Now let us set

$$
\tau_{m}=\exp \left(-t^{3} / 24\right) T_{m}(t)
$$

It follows from the Toda equation (4.3) with $c(m)=1$ that

$$
\left\{\begin{aligned}
T_{m+1}(t) & =\left(T_{m}(t)^{2} t-4\left(T_{m}^{\prime \prime}(t) T_{m}(t)-T_{m}^{\prime}(t)^{2}\right)\right) / T_{m-1}(t) \\
T_{0}(t) & =T_{1}(t)=1 .
\end{aligned}\right.
$$

We find here the Yablonskii-Vorob'ev polynomials. It follows immediately from (4.3) that the $T_{m}(t)$ 's are rational functions. In Section 5 , we will discuss proofs that the $T_{m}(t)$ 's are in fact polynomials. It follows from the definition of $q(b-1), p(b-1)$ we have

$$
H(b-1, t, q(b-1), p(b-1))=H(b, t, q(b), p(b))+q(b) .
$$

Therefore by (4.1), (4.3) and (4.5) we get

$$
\tau_{m+1}^{\prime} / \tau_{m+1}-\tau_{m}^{\prime} / \tau_{m}=q\left(-\frac{1}{2}-m\right) .
$$

So (4.6) shows that the Yablonskii-Vorob'ev polynomials give an effective method of calculating the rational solution $q\left(-\frac{1}{2}-m\right)$ of the second Painlevé equation $P_{2}(-m-1): q^{\prime \prime}=2 q^{3}+t q-(m+1)$ for $m \in \mathbb{Z}$.

The conclusion is that the recurrence relation (2.2) comes form the Toda equation satisfied by the $\tau$-functions.

\section{Proof that the $T_{m}(t)$ 's are polynomials}

The quickest way of proving that the $T_{m}(t)$ 's are polynomials is to use the following fundamental

Theorem 5.1. - For the second Painlevé equation, a $\tau$-function $\tau(t)$ is holomorphic over $\mathbb{C}$.

If we admit the Theorem, since $\tau_{m}=T_{m}(t) \exp \left(-t^{3} / 24\right)$, the rational function $T_{m}(t)$ is holomorphic over $\mathbb{C}$ and hence $T_{m}(t)$ is a polynomial. Theorem 5.1 is a deep and transcendental result, which we would like to avoid for the following reason. When we want to generalize the Yablonskii-Vorob'ev 
polynomials to the other Painlevé equations, the analogous theorems for the other Painlevé equations do not allows us to prove that the rational functions in question are polynomials but they show only that the rational functions are regular over $\mathbb{C} \backslash\{0,1\}$ for example if we deal with the $P_{6}$. The best way of showing that the $T_{m}(t)$ 's are polynomials is as follows. As we noticed above, the $T_{m}(t)$ 's are rational functions by the recurrence relation (4.4). So $\tau_{m}=T_{m} \exp \left(-t^{3} / 24\right)$ is meromorphic over $\mathbb{C}$. Consequently $\tau_{m}^{\prime} / \tau_{m}=$ $H\left(-\frac{1}{2}-m, t, q\left(-\frac{1}{2}-m\right), p\left(-\frac{1}{2}-m\right)\right)=h\left(-\frac{1}{2}-m, q\left(-\frac{1}{2}-m\right), p\left(-\frac{1}{2}-m\right)\right)$ is meromorphic over $\mathbb{C}$ and satisfy the differential equation $E\left(-\frac{1}{2}-m\right)$. We can easily show

LEMMA 5.2. - If a solution $h$ of $E\left(-\frac{1}{2}-m\right)$ is meromorphic at a point $a \in \mathbb{C}$, then $t=a$ is at most a pole of order 1 . Moreover if the point a is a pole of order 1 of $h$, then the residue is equal to 1.

Now Lemma 5.2 shows that $\tau_{m}$ is regular over $\mathbb{C}$ so that $T_{m}(T)$ is a polynomial.

\section{Special polynomials}

We apply the method of the previous sections to the Painleve equations $P_{j}(3 \leqslant j \leqslant 6)$. As we illustrated in Section 3 taking the second equation as an example, the Painlevé equation $P_{j}$ has a Hamiltonian representation

$$
S_{j}(\mathbf{v}) \quad\left\{\begin{array}{l}
\mathrm{d} q / \mathrm{d} t=\partial H_{j} / \partial p, \\
\mathrm{~d} p / \mathrm{d} t=-\partial H_{j} / \partial q,
\end{array}\right.
$$

where $H_{j}=H_{j}(\mathbf{v}, t, q, p)$ is a polynomial of $q, p$ and $\mathbf{v}$ with coefficients in $\mathbb{Q}(t)$. Namely $S_{j}(\mathbf{v})$ is a Hamiltonian system parametrized by $\mathbf{v}$ which belongs to a complex vector space $V_{j}$. An appropriate extended Weyl group $G_{j}$ operates not only on the parameter space $V_{j}$ but also on the set of solutions of the system $S_{j}(\mathbf{v})$.

Remark 6.1. - Precisely speaking, we must enlarge $G_{j}$ to $\widetilde{G}_{j}$ or we must consider an extension $\widetilde{G}_{j}$ of the group $G_{j}$ so that $\widetilde{G}_{j}$ operates on the set of solutions of $S_{j}(\mathbf{v})$. Namely we can find a group $\widetilde{G}_{j}$ such that $G_{j}$ is a quotient group of $G_{j}$ and such that $G_{j}$ operates on the set of solutions of the system $S_{j}(\mathbf{v})$ as well as on the parameter space $V_{j}$. We know, however, $\widetilde{G}_{j}=G_{j}$ for $j=2,4$. 
6.1. The fourth Painlevé equation. The fourth equation

$$
P_{4}(\alpha, \beta) \quad y^{\prime \prime}=\frac{y^{\prime 2}}{2 y}+\frac{3 y^{3}}{2}+4 t y^{2}+2\left(t^{2}-\alpha\right) y+\frac{\beta}{y}
$$

is equivalent to the Hamiltonian system

$$
S_{4}\left(v_{1}, v_{2}, v_{3}\right) \quad\left\{\begin{array}{l}
\mathrm{d} q / \mathrm{d} t=4 p q-q^{2}-2 t q+2\left(v_{1}-v_{2}\right), \\
\mathrm{d} p / \mathrm{d} t=-2 p^{2}+2 q+2 t+\left(v_{1}-v_{3}\right),
\end{array}\right.
$$

with Hamiltonian $H_{4}=2 q p^{2}-\left(q^{2}+2 t q-2\left(v_{1}-v_{2}\right)\right) p-\left(v_{1}-v_{3}\right) q$, where $\left(v_{1}, v_{2}, v_{3}\right) \in \mathbb{C}^{3}$ belongs to the paprameter space $V_{4}=\left\{\left(v_{1}, v_{2}, v_{3}\right) \in \mathbb{C}^{3} \mid\right.$ $\left.v_{1}+v_{2}+v_{3}=0\right\}$ (cf. [3, 7]). Here the parameters $v_{1}, v_{2}, v_{3}$ are related with the usual parameters $\alpha, \beta$ of the Painlevé equation $P_{4}(\alpha, \beta)$ by $\alpha=$ $3 v_{3}+1, \beta=-2\left(v_{1}-v_{2}\right)^{2}$. The extended affine Weyl group of type $A_{2}$ operates on both the parameter space $V_{4}$ and the set of solutions of the system $S_{4}\left(v_{1}, v_{2}, v_{3}\right)$. We consider a translation $v \mapsto v+\frac{1}{3}(-1,-1,2)$ and a permutation $\left(v_{1}, v_{2}, v_{3}\right) \mapsto\left(v_{3}, v_{1}, v_{2}\right)$, which are affine transformations of the parameter space and belong to the extended affine Weyl group. Their composite is the affine transformation $\left(v_{1}, v_{2}, v_{3}\right) \rightarrow\left(v_{3}+2 / 3, v_{1}-1 / 3, v_{2}-1 / 3\right)$ so that $\frac{1}{3}(1,0,-1)$ is the fixed point. Let $(q(t), p(t))$ be a solution of $S_{4}(1 / 3,0,-1 / 3)$. Let $h=H_{4}((1 / 3,0,-1 / 3), t, q(t), p(t))$. Then the argument of Lemma 3.16 shows $(q(t), p(t))$ is fixed by the transformation $\mathbf{v} \mapsto \mathbf{v}+\frac{n}{3}(-1,-1,2)$ if and only if $h+q+2 t / 3=h$ or equivalently $q=-2 t / 3$. If $q=-2 t / 3$, then $p=$ $t / 3+1 /(2 t)$ by the first equation of $S_{4}(1 / 3,0,-1 / 3)$. We discover the fixed solution $(q(t), p(t))=(-2 t / 3, t / 3+1 /(2 t))$ at $\mathbf{v}=\frac{1}{3}(1,0,-1)$. Substituting the solution into the Hamiltonian, we get $H_{4}(1 / 3(1,0,-1), t, q(t), p(t))=$ $4 t^{3} / 27+2 t / 3$ and the $\tau$-function $\tau_{0}(t)$ satisfies $\tau_{0}^{\prime}(t) / \tau_{0}(t)=4 t^{3} / 27+2 t / 3$ so that $\tau_{0}=$ const. $\exp \left(\int\left(4 t^{3} / 27+2 t / 3\right) \mathrm{d} t\right)=$ const. $\exp \left(t^{4} / 27+t^{2} / 3\right)$. We consider the solutions translated by the above translations $v \mapsto v+\frac{n}{3}(-1,-1,2)$ with $n \in \mathbb{Z}$ as well as their $\tau$-functions $\tau_{n}(t)$. It follows from the Toda equation ([3, Formula (3.18), p. 242])

$$
\mathrm{d}^{2} \log \tau_{n} / \mathrm{d} t^{2}+2(n-2 / 3)=c(n) \tau_{n-1} \tau_{n+1} / \tau_{n}^{2}
$$

with $c(n)=1$ that we have

$$
T_{n+1}(t)=\left(T_{n}^{\prime \prime}(t) T_{n}(t)-T_{n}^{\prime}(t)^{2}+\left(4 t^{2} / 9+2(2 n-1) / 3\right) T_{n}(t)^{2}\right) / T_{n-1}(t)
$$

with $T_{0}(t)=T_{1}(t)=1$. If we set $x=\sqrt{3 / 2 t}$ and $T_{n}(t)=S_{n}(x)$ with $c(m)=2 / 3$, we get

$$
S_{n+1}(x)=\left(S_{n}^{\prime \prime}(x) S_{n}(x)-S_{n}^{\prime}(x)^{2}+\left(x^{2}+(2 n-1)\right) S_{n}(x)^{2}\right) / S_{n-1}(x),
$$

with $S_{0}(x)=S_{1}(x)=1 . S_{n}(x)$ is a polynomial of degree $n(n-1)$, which is called the Okamoto polynomial (cf. 8.2). The Okamoto polynomials appear at the barycenters $\frac{1}{3}(1-k,-k, 2 k-1), k \in \mathbb{N}$ of the alcoves, whereas the Hermite functions are on the wall $v_{1}-v_{3}=0$. In particular the Hermite 
polynomial appear at the points $\frac{k}{3}(-1,2,-1), k=0,1,2, \ldots$ We notice here that the reflection with respect to a wall induces the identity transformation of the solutions on the wall as for $P_{2}$.

6.2. The third Painlevé equation. The third equation

$$
P_{3}(\alpha, \beta, \gamma, \delta) \quad y^{\prime \prime}=\frac{y^{\prime 2}}{y}-\frac{y^{\prime}}{t}+\frac{\alpha y^{2}+\beta}{t}+\gamma y^{3}+\frac{\delta}{y}
$$

is equivalent to the Hamiltonian system

$$
S_{3}\left(v_{1}, v_{2}\right) \quad\left\{\begin{array}{l}
t \mathrm{~d} q / \mathrm{d} t=2 q^{2} p-q^{2}-v_{1} q+t \\
t \mathrm{~d} p / \mathrm{d} t=-2 q p^{2}+2 q p+v_{1} p-\left(v_{1}+v_{2}\right) / 2
\end{array}\right.
$$

with Hamiltonian $H_{3}(\mathbf{v}, t, q, p)=\left(q^{2} p^{2}-\left(q^{2}+v_{1} q-t\right) p+\frac{1}{2}\left(v_{1}+v_{2}\right) q\right) / t$, where $\left(v_{1}, v_{2}\right)$ belongs to the parameter space $\mathbb{C}^{2}$ (cf. $\left.[6,7]\right)$. In this case the equivalence is exceptional. Namely we replace $t$ in the third equation $P_{3}(\alpha, \beta, \gamma, \delta)$ by $t_{1}$ and introduce $t$ by $t_{1}^{2}=t$. Then $S_{3}(\mathbf{v})$ is equivalent to $P_{3}(\alpha, \beta, \gamma, \delta)$. Namely after the field extension $\mathbb{Q}(t) \rightarrow \mathbb{Q}\left(t_{1}\right), S_{3}(\mathbf{v})$ is equivalent to $P_{3}(\alpha, \beta, \gamma, \delta)$. Here we normalized $\gamma=4, \delta=-4$ and $v_{1}, v_{2}$ and $\alpha, \beta$ are related by $\alpha=-4 v_{2}, \beta=4\left(v_{1}+1\right)$. The corresponding Lie algebra is $B_{2}$. We consider a translation $\left(v_{1}, v_{2}\right) \mapsto\left(v_{1}+1, v_{2}+1\right)$ and the reflection with respect to a line $v_{1}+v_{2}=0$. So their composite is the reflection with respect to a line $v_{1}+v_{2}+1=0$. Let $(q(t), p(t))$ be a solution of $S_{4}\left(v_{1}, v_{2}\right)$ with $v_{1}+v_{2}+1=0$. We set $h=t H_{3}\left(v_{1}, v_{2}, t, q(t), p(t)\right)+v_{1}^{2} / 4-t / 2$. Then the argument of Lemma 3.6 shows that the solution $(q(t), p(t))$ is fixed by the reflection with respect to the line $v_{1}+v_{2}+1=0$ if and only if $h+\left(2 v_{1}+1\right) / 4-q(t)(p(t)-1)=h$ or equivalently

$$
q(t)(p(t)-1)=\frac{2 v_{1}+1}{4} .
$$

Differentiating (6.3.1) and eliminating $q^{\prime}, p^{\prime}(t)$ by using $S_{3}\left(v_{1}, v_{2}\right)$, we get

$$
q+2 q^{2}-2 p q^{2}-2 t+2 p t+2 q v_{1}=0 .
$$

Eliminating $p$ from (6.2.1) and (6.2.2), we get

$$
\left(1+2 v_{1}\right)\left(q^{2}+t\right)=0
$$

We have either $v_{1}=-\frac{1}{2}$ or $q^{2}=-1$. In the first case, it follows from (6.2.2) that we have $q(t)=0$ or $p(t)=1$. Since $q=0$ contradicts the first equation of system $S\left(v_{1}, v_{2}\right)$, we conclude $p(t)=1$. Then it follows from the first equation of system $S\left(v_{1}, v_{2}\right) t \mathrm{~d} q / \mathrm{d} t=2 q^{2}-q^{2}-v_{1} q+t$ so that we find a classical solution $(q, p)=(q, 1)$, where $q$ satisfies $t \mathrm{~d} q / \mathrm{d} t=$ $2 q^{2}-q^{2}-v_{1} q+t$. Since we are looking for a fixed algebraic solution, let us study the second case $q^{2}=-t$. Then it follows from (6.2.1) $p=$ $1+\left(2 v_{1}+1\right) /(4 q)$. In other words, we find the fixed solution $(q(t), p(t))=$ 
$\left(s, 1+\left(1+2 v_{1}\right) /(4 s)\right)$, which is algebraic, on the line $v_{1}+v_{2}+1=0$, where $s^{2}=-t$. So we assume from now on $v_{1}+v_{2}+1=0$. Substituting the solution into the Hamiltonian, we get $H_{3}\left(v_{1}, v_{2}, t, q(t), p(t)\right)=1-\left(v_{1}+\frac{1}{2}\right) s t^{-1}+$ $\left(\left(1+2 v_{1}\right)\left(1-2 v_{1}\right) / 16\right) t^{-1}$. We consider the translations by $n(1,1)(n \in \mathbb{N})$ of the fixed solution on the line $v_{1}+v_{2}+1=0$ and their $\tau$-functions $\tau_{n}(t)$. We set $\tau(t)=T_{n}(t) \exp \left(\int\left(H_{3}\left(v_{1}, v_{2}, t, q(t), p(t)\right)-\frac{n}{4}\left(2 v_{1}+1\right) t^{-1}\right) \mathrm{d} t\right)$. Then it follows from the Toda equation ([6, Formula (3.12), p. 328])

$$
\frac{\mathrm{d}}{\mathrm{d} t} t \frac{\mathrm{d}}{\mathrm{d} t} \log \tau_{n}=c(n) \frac{\tau_{n-1} \tau_{n+1}}{\tau_{n}^{2}}
$$

with $c(n)=1$ that we get

$$
T_{n+1}(s)=\frac{-\left(\frac{\mathrm{d} T_{n}(s)}{\mathrm{d} s}+s \frac{\mathrm{d}^{2} T_{n}(s)}{\mathrm{d} s^{2}}\right) T_{n}(s)+s \frac{\mathrm{d} T_{n}(s)^{2}}{\mathrm{~d} s}+\left(4 s+2 v_{1}+1\right) T_{n}(s)^{2}}{4 s T_{n-1}(s)}
$$

with $T_{0}(s)=T_{1}(t)=1$. The polynomials $T_{n}(s)$ 's contain the parameter $v_{1}$ so that we may denote $T_{n}(s)$ by $T_{n}\left(v_{1}, s\right)$. If we set $u=1 /(4 s)$ and $S_{n}(a, u)=T_{n}\left(v_{1}, s\right)$ and $a=2 v_{1}+1$, we get

$$
\begin{aligned}
& S_{n+1}(a, u) \\
& =\frac{-\left(4 u^{3} \frac{\partial S_{n}(a, u)}{\partial u}+4 u^{4} \frac{\partial^{2} S_{n}(a, u)}{\partial u^{2}}\right) S_{n}(a, u)+4 u^{4} \frac{\partial S_{n}(a, u)^{2}}{\partial u}+(1+a u) S_{n}(a, u)^{2}}{S_{n-1}(a, u)} .
\end{aligned}
$$

The general theory of the $\tau$-function tells us that $\tau_{n}(t)$ is regular over the universal covering space of $\mathbb{C} \backslash\{0\}$ so that $S_{n}(u)$ is a Laurent polynomial of $u$. We can show, however, that $S_{n}(a, u)$ is a polynomial of $a$ and $u$ such that $\operatorname{deg}_{u} S_{n}(a, u)=n(n-1) / 2$ (cf. (8.3)).

6.3. The fifth Painlevé equation. The fifth equation

$$
P_{5}(\alpha, \beta, \gamma, \delta) \quad y^{\prime \prime}=\left(\frac{1}{2 y}+\frac{1}{y-1}\right) y^{\prime 2}-\frac{y^{\prime}}{t}+\frac{(y-1)^{2}}{t^{2}}\left(\alpha y+\frac{\beta}{y}\right)+\frac{\gamma y}{t}+\delta \frac{y(y+1)}{y-1}
$$

is equivalent to the Hamiltonian system

$$
S_{5}(\mathbf{v})\left\{\begin{array}{r}
t \mathrm{~d} q / \mathrm{d} t=2 q(q-1)^{2} p+\left(v_{1}-v_{2}\right)(q-1)^{2}+2\left(v_{1}+v_{2}\right) q(q-1)-t q, \\
t \mathrm{~d} p / \mathrm{d} t=-\left(3 q^{2}-4 q+1\right) p^{2}-\left(\left(6 v_{1}+2 v_{2}\right) q-4 v_{1}-t\right) p \\
-\left(v_{1}-v_{3}\right)\left(v_{1}-v_{4}\right),
\end{array}\right.
$$

the Hamitonian $H_{5}$ being

$$
\begin{aligned}
\frac{q(q-1)^{2} p^{2}+\left(\left(v_{1}-v_{2}\right)(q-1)^{2}+2\left(v_{1}+v_{2}\right) q(q-1)-t q\right) p}{t} & \\
+ & \frac{\left(v_{1}-v_{3}\right)\left(v_{1}-v_{4}\right)(q-1)}{t},
\end{aligned}
$$


where $\left(v_{1}, v_{2}, v_{3}, v_{4}\right)$ belongs to the parameter space $\left\{\mathbf{v}=\left(v_{1}, v_{2}, v_{3}, v_{4}\right) \in \mathbb{C}^{4} \mid\right.$ $\left.v_{1}+v_{2}+v_{3}+v_{4}=0\right\}$. Here we normalize $\delta=-\frac{1}{2}$, and the parameters $v_{i}$ 's and $\alpha, \beta, \gamma$ are related by $\alpha=\left(v_{3}-v_{4}\right)^{2} / 2, \beta=-\left(v_{1}-v_{2}\right)^{2} / 2, \gamma=$ $2\left(v_{1}+v_{2}+1\right)$. The corresponding Lie algebra is $A_{3}$. So the extended affine Weyl group of type $A_{3}$ operates on the parameter space. We consider the translation $\mathbf{v} \mapsto \mathbf{v}+1 / 4(1,-3,1,1)$ and a permutation (1432) that naturally operates on the parameter space. These are operations of elements of the extended affine Weyl group. Their composite $f$ sends $\mathbf{v}=\left(v_{1}, v_{2}, v_{3}, v_{4}\right)$ to $\left(v_{2}-3 / 4, v_{3}+1 / 4, v_{4}+1 / 4, v_{1}+1 / 4\right)$. The transformation $f$ is of order 4 and $1 / 8(-3,3,1,-1)$ is the fixed point. The fixed solution at the fixed point and its translations by $n / 4(1,-3,1,1), \boldsymbol{n} \in \mathbb{N}$ give special polynomials but $f^{2}$ yields more general and interesting polynomials involving a parameter. Namely the set of fixed points of $f^{2}$ is a line $\left\{\left(v, \frac{1}{2}-v, v-\frac{1}{2},-v\right) \in \mathbb{C}^{3} \mid v \in \mathbb{C}\right\}$ of the parameter space. The fixed solution of the Hamiltonian system on this line is $(q(t), p(t))=(-1, t / 8+v)$ which contains a parameter $v$. Substituting $(p(t), q(t))$ in the Hamiltonian, we get $H_{5}\left(v, \frac{1}{2}-v, v-\frac{1}{2},-v ; t, q(t), p(t)\right)=$ $t / 16+v+2 v(2 v-1) / t$ so that $\tau_{0}=\exp \left(\int(t / 16+v+2 v(2 v-1) / t) \mathrm{d} t\right)=$ $2 v(2 v-1) \exp \left(t^{2} / 32+v t\right)$. Let $\tau_{m}$ be a $\tau$-function of the solution obtained from the $\operatorname{solution}(q(t), p(t))$ of $S_{5}\left(v, \frac{1}{2}-v, v-\frac{1}{2},-v\right)$ by the translation $\mathbf{v} \mapsto \mathbf{v}+n / 4(1,-3,1,1)$.

We set $\tau_{n}(t)=T_{n}(t) \exp \left(\int(t / 16+v+2 v(2 v-1) t-n(t / 4-2 v-1) / t) \mathrm{d} t\right.$. Then it follows from the Toda equation

$$
\frac{\mathrm{d}}{\mathrm{d} t} t \frac{\mathrm{d}}{\mathrm{d} t} \log \tau_{n}+v_{4}-v_{1}+n=c(n) \frac{\tau_{n-1} \tau_{n+1}}{\tau_{n}^{2}}
$$

$([5$, p. 68 , Formula $(2.9)])$

$$
T_{n+1}=\left((t / 8-v+3 n / 4) T_{n}^{2}+T_{n}^{\prime} T_{n}+t T_{n}^{\prime \prime} T_{n}-t T_{n}^{2}\right) / T_{n-1}
$$

with $T_{0}=T_{1}=1$. The general theory of the $\tau$-functions shows that $T_{n}(v, t)$ is a Laurent polynomial of $t$. We can, however, prove that $T_{n}(v, t)$ is a polynomial in $t$ and $v$ of degree $n(n-1) / 2$ (cf. 8.4).

6.4. The sixth Painlevé equation. The sixth equation

$$
\begin{aligned}
q^{\prime \prime}=\frac{1}{2}\left(\frac{1}{q}+\frac{1}{q-1}+\frac{1}{q-t}\right) q^{\prime 2}- & \left(\frac{1}{t}+\frac{1}{t-1}+\frac{1}{q-t}\right) q^{\prime} \\
+\frac{q(q-1)(q-t)}{t^{2}(t-1)^{2}} & \left(\alpha+\beta \frac{t}{q^{2}}+\gamma \frac{t-1}{(q-1)^{2}}+\delta \frac{t(t-1)}{(q-t)^{2}}\right) \\
- & 1078-
\end{aligned}
$$


is equivalent to the Hamiltonian system

$$
S_{6}(\mathbf{b})\left\{\begin{array}{l}
\frac{t \mathrm{~d} q}{\mathrm{~d} t}=\frac{\partial H_{6}}{\partial p} \\
\frac{t \mathrm{~d} p}{\mathrm{~d} t}=-\frac{\partial H_{6}}{\partial q}
\end{array}\right.
$$

with Hamiltonian $H_{6}(\mathbf{b}, t, q, p)=\left[q(q-1)(q-t) p^{2}-\left\{\left(b_{1}+b_{2}\right)(q-1)(q-t)+\right.\right.$ $\left.\left.\left(b_{1}-b_{2}\right) q(q-t)+\left(b_{3}+b_{4}\right) q(q-1)\right\} p+\left(b_{1}+b_{3}\right)\left(b_{1}+b_{4}\right)\right] /(t(t-1))$, where $\left(b_{1}, b_{2}, b_{3}, b_{4}\right)$ belongs to the parameter space $\mathbb{C}^{4}$. The parameters are related by $\alpha=\left(b_{3}-b_{4}\right)^{2} / 2, \beta=-\left(v_{1}+b_{2}\right)^{2} / 2, \gamma=\left(b_{1}-b_{2}\right)^{2} / 2, \delta=-\left(b_{3}+b_{4}\right)^{2} / 2$. The corresponding Lie algebra is $D_{4}$. We consider a translation $\mathbf{v} \mapsto \mathbf{v}+$ $(0,0,1,0)$ and a linear map $\left(b_{1}, b_{2}, b_{3}, b_{4}\right) \mapsto\left(b_{1}, b_{2},-b_{3},-b_{4}\right)$, which are operations of elements of the extended Weyl group of the Lie algebra of type $D_{4}$. Their composite is $\left(b_{1}, b_{2}, b_{3}, b_{4}\right) \mapsto\left(b_{1}, b_{2},-b_{3}-1,-b_{4}\right)$ and hence $\left(b_{1}, b_{2},-\frac{1}{2}, 0\right)$ is the fixed point. We look for fixed solutions for $\left(b_{1}, b_{2},-\frac{1}{2}, 0\right)$. To this end, let us set according to [4] $h(\mathbf{b}, t)=H_{6}(\mathbf{b}, t, q(t), p(t))+\sigma_{2}^{\prime}[\mathbf{b}] t-$ $\sigma_{2}[\mathbf{b}]$ for a solution $(q(t), p(t))$ of $S_{6}(\mathbf{v})$, where $\sigma_{2}^{\prime}$ is the second symmetric polynomial of $b_{1}, b_{3}, b_{4}$ and $\sigma_{2}[\mathbf{b}]$ is the second symmetric polynomial of $b_{1}, b_{2}, b_{3}, b_{4}$. We denote $h(\mathbf{b}, t)-q(t)(q(t)-1) p(t)+\left(b_{1}+b_{4}\right) q(t)-\left(b_{1}+b_{2}+\right.$ $\left.b_{4}\right) / 2$ by $h^{+}(\mathbf{b}, t)$. Then it follows from [4, Formula (1.13), p. 352] a solution $(q(t), p(t))$ of $S_{6}\left(b_{1}, b_{2},-\frac{1}{2}, 0\right)$ is fixed by the above transformation if and only if $h^{+}\left(b_{1}, b_{2},-\frac{1}{2}, 0\right)=h\left(b_{1}, b_{2},-\frac{1}{2}, 0\right)$ or equivalently

$$
-q(t)(q(t)-1) p(t)+b_{1} q(t)-\frac{1}{2}\left(b_{1}+b_{2}\right)=0
$$

by definition. Differentiating (6.4.1) and eliminating $q^{\prime}(t), p^{\prime}(t)$ by using the system $S_{6}\left(b_{1}, b_{2},-\frac{1}{2}, 0\right)$, we get

$$
\begin{array}{r}
-p^{2} q^{3}+p^{2} q^{4}-p^{2} q t+3 p^{2} q^{2} t-2 p^{2} q^{3} t+p q^{2} b_{1}-2 p q^{3} b_{1}+p t b_{1} \\
-4 p q t b_{1}+4 p q^{2} t b_{1}+q^{2} b_{1}^{2}+t b_{1}^{2}-2 q t b_{1}^{2}+p q^{2} b_{2}+p t b_{2} \\
-2 p q t b_{2}-q b_{1} b_{2}+t b_{1} b_{2}=0 .
\end{array}
$$

It follows from (6.4.1)

$$
p(t)=\frac{1}{q(t)(q(t)-1)}\left(b_{1} q(t)-\frac{1}{2}\left(b_{1}+b_{2}\right)\right) .
$$

Substituting (6.4.3) into (6.4.2), we get

$$
\left(-b_{1}^{2}+2 b_{1} b_{2}-b_{2}^{2}-4 t b_{1} b_{2}\right) q^{2}+2 t\left(b_{1}+b_{2}\right)^{2} q-t\left(b_{1}+b_{2}\right)^{2}=0 .
$$

So we have

$$
\begin{gathered}
q(t)=\frac{t\left(b_{1}+b_{2}\right)^{2}+\left(b_{1}+b_{2}\right)\left(b_{1}-b_{2}\right) \sqrt{t(t-1)}}{\left(b_{1}-b_{2}\right)^{2}+4 t b_{1} b_{2}} \\
-1079-
\end{gathered}
$$


and consequently

$$
p(t)=\frac{b_{1} q-\frac{1}{2}\left(b_{1}+b_{2}\right)}{q(q-1)}
$$

by (6.4.3). Substituting $(q(t), p(t))$ into the Hamiltonian $H_{6}$, we get

$$
\begin{aligned}
H_{6}\left(b_{1}, b_{2},-\frac{1}{2}, 0 ; t, q(t), p(t)\right) & \\
=-b_{1}+2 t b_{1} & +b_{1}^{2}-2 t b_{1}^{2}+2 \sqrt{t(t-1)} b_{1}^{2} \\
& \quad-b_{2}+2 b_{1} b_{2}+b_{2}^{2}-2 t b_{2}^{2}-\frac{2 \sqrt{t(t-1)} b_{2}^{2}}{4 t(t-1)},
\end{aligned}
$$

which we denote by $\mathcal{H}_{0}$. So we have $\tau_{0}=\exp \left(\int \mathcal{H}_{0} \mathrm{~d} t\right)$. Let $\tau_{n}$ be a $\tau$-function of the solution of $S_{6}\left(b_{1}, b_{2},-\frac{1}{2}+n, 0\right)$ obtained from the solution $(q(t), p(t))$ by the translation $\mathbf{v} \mapsto \mathbf{v}+n(0,0,1,0)$. We set

$$
\tau_{n}(t)=T_{n}(t) \exp \left(\int\left(\mathcal{H}_{0}-\frac{n\left(b_{1} t-\frac{1}{2}\left(b_{1}+b_{2}\right)\right)}{t(t-1)}\right) \mathrm{d} t\right) .
$$

Then it follows from the Toda equation

$$
\frac{\mathrm{d}}{\mathrm{d} t} t(t-1) \frac{\mathrm{d}}{\mathrm{d} t} \log \tau_{n}+\left(b_{1}+b_{3}+n\right)\left(b_{3}+b_{4}+n\right)=c(n) \frac{\tau_{n-1} \tau_{n+1}}{\tau_{n}^{2}}
$$

with $c(n)=1$ (cf. [4, Formula (4.4), p. 368]),

$$
\begin{aligned}
& T_{n+1}=\frac{\left\{\frac{1}{4}\left(-2 b_{1}^{2}-2 b_{2}^{2}+\left(b_{1}^{2}-b_{2}^{2}\right) \frac{2 t-1}{\sqrt{t(t-1)}}\right)+\left(n-\frac{1}{2}\right)^{2}\right\} T_{n}^{2}}{T_{n-1}} \\
& +\frac{(2 t-1) T_{n}^{\prime} T_{n}+t(t-1)\left(T_{n}^{\prime \prime} T_{n}-T_{n}^{\prime 2}\right)}{T_{n-1}} .
\end{aligned}
$$

Now we introduce a new variable $v$ by $v=\sqrt{t /(t-1)}+\sqrt{(t-1) / t}$. Then the recurrence formula (6.5) is translated into

$$
\begin{aligned}
T_{n+1}= & \frac{\left\{\frac{1}{4}\left(-2 b_{1}^{2}-2 b_{2}^{2}+\left(b_{1}^{2}-b_{2}^{2}\right) v\right)+\left(n-\frac{1}{2}\right)^{2}\right\} T_{n}^{2}}{T_{n-1}} \\
& +\frac{\frac{1}{4}\left(v^{2}-4\right)\left\{\left(v^{2}-4\right) \frac{\mathrm{d}^{2} T_{n}}{\mathrm{~d} v^{2}}+v \frac{\mathrm{d} T_{n}}{\mathrm{~d} v}\right\} T_{n}-\left(\frac{1}{2}\left(v^{2}-4\right) \frac{\mathrm{d} T_{n}}{\mathrm{~d} v}\right)^{2}}{T_{n-1}} .
\end{aligned}
$$

Since $T_{n}(v)$ contains parameters $b_{1}, b_{2}$, we denote it by $T\left(b_{1}, b_{2}, v\right)$. We can show that $T_{n}\left(b_{1}, b_{2}, v\right)$ is a polynomial in $b_{1}, b_{2}, v$ and $\operatorname{deg}_{v} T_{n}=n(n-1) / 2$ (see 8.5). 


\section{Special polynomials and Young diagrams}

We do not know any significance of our polynomials. Attending at our talk at Montreal, a weekend is enough for Noumi and Okamoto to reveal a hidden structure of our polynomials for $P_{6}$.

7.1. Polynomials in (6.4) for $P_{6}$. We already need several pages to print the polynomial $T_{5}\left(b_{1}, b_{2}, v\right)$ of Section 6 for $P_{6}$. Let $z=(2-v) / 4$, $w=(2+v) / 4, d=-4 b_{1}^{2}, c=-4 b_{2}^{2}$ and

$$
\begin{aligned}
& d_{n}:=\left(d+1^{2}\right) z \cdot\left(d+3^{2}\right) z \cdots\left(d+(2 n-1)^{2}\right) z, \\
& c_{n}:=\left(c+1^{2}\right) w \cdot\left(c+3^{2}\right) w \cdots\left(c+(2 n-1)^{2}\right) w
\end{aligned}
$$

for $n \in \mathbb{N}$. Denoting $2^{n(n-1)} T_{n}\left(b_{1}, b_{2}, u\right)$ by $U_{n}(w, z)$, they observed $U_{2}=$ $d_{1}+c_{1}, \quad U_{3}=d_{1} d_{2}+3 c_{1} d_{2}+3 c_{2} d_{1}+c_{1} c_{2}, \quad U_{4}=d_{1} d_{2} d_{3}+6 c_{1} d_{2} d_{3}+$ $15 c_{2} d_{1} d_{3}+10 c_{1} c_{2} d_{3}+10 c_{3} d_{1} d_{2}+15 c_{1} c_{3} d_{2}+6 c_{2} c_{3} d_{1}+c_{1} c_{2} c_{3}$. Here it is convenient to attach a pair of Young diagrams

$$
(\emptyset, \square)
$$

for $d_{1}$,

$$
(\square, \emptyset)
$$

for $c_{1}$,

$$
(\emptyset, \square)
$$

for $d_{1} d_{2}, \ldots$, etc. so that for example the corresponding pair for $c_{1} d_{2} d_{3}$ is

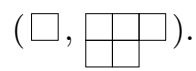

So

$$
\begin{aligned}
U_{4}= & (\emptyset, \square)+6(\square, \square)+15(\square, \square,+10(\square \square, \square) \\
& +10(\square, \square)+15(\square \square, \square)+6(\square, \square)+(\square, \emptyset) .
\end{aligned}
$$

Here two monomials with coefficients 10 give the coefficients of $w^{3} z^{3}$. The question is whether the $U_{n}$ 's have such expressions for every $n \in \mathbb{N}$ and if so how the coefficients are determined. For example, in $U_{4}$ the binomial coefficients $(1,6,15,20,15,6,1)$ appear. Why 20 is divided into 10 for

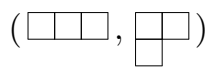

and 10 for

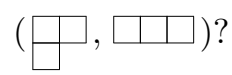


In this case, $20=10+10$ is reasonable because two diagrams are dual and because the polynomial is symmetric for the permutation of $(d, w)$ and $(c, z)$ but we have a non-trivial decomposition say in $U_{5}: 120$ is divided into 50 for

and 70 for
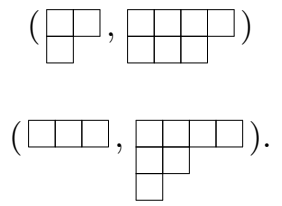

A thorough investigation on this decomposition will be done in [2].

Noumi noticed further the constant terms of the Okamoto polynomials have simple factorizations. Let $S_{n}(x)$ be the Okamoto polynomials in 6.1. Then we have $S_{0}(0)=S_{1}(0)=S_{2}(0)=1, S_{3}(0)=5, S_{4}(0)=5^{2} .7$, $S_{5}(0)=5^{3} 7^{2} 11, S_{6}(0)=5^{4} 7^{3} 11^{2} 13, \quad S_{7}(0)=5^{5} 7^{4} 11^{3} 13^{2} .17, \quad S_{8}(0)=$ $5^{6} 7^{5} 11^{4} 13^{3} 17^{2} 19, \ldots$ So looking at the exponents, we have a feeling that they are related to the Young diagram too. The constant terms of the Yablonskii-Vorob'ev polynomials have similar factorizations. Namely let $T_{n}(t)$ be the Yablonskii-Vorob'ev polynomial. We have $T_{2}(0)=2^{2}, T_{3}(0)=$ $-2^{4} 5, \quad T_{4}(0)=0, T_{5}(0)=-2^{10} 5^{3} 7^{2}, T_{6}(0)=-2^{14} 5^{4} 7^{3} 11, \quad T_{7}(0)=0$, $T_{8}(0)=-2^{24} 5^{6} 7^{5} 11^{3} 13^{2}, T_{9}(0)=2^{30} 5^{7} 7^{6} 11^{4} 13^{3} 17, \ldots$

7.2. Our polynomials for $P_{3}$ and $P_{5}$ resemble and they look also related with the Young diagrams. To illustrate this, let us take our polynomials $T_{n}(v, t)$ of $(6.3)$ for $P_{5}$. It is convenient to introduce $l(i):=(i-4 v) / 4$. Their constant terms $T_{n}(v, 0)$ are $1,1, l(3), l(3) l(4) l(5), l(3) l(4) l(5)^{2} l(6) l(7)$, $l(3) l(4) l(5)^{2} l(6)^{2} l(7)^{2} l(8) l(9), \ldots$ Moreover if we set $z=t / 8$, we can interpret a first few of them as $T_{0}(v, t)=T_{1}(v, t)=1, T_{2}(v, t)=z+l(3)$, $T_{3}(v, t)=z^{3}+3 l(4) z^{2}+3 l(3) l(5) z+l(3) l(4) l(5), T_{4}(v, t)=z^{6}+6 l(5) z^{5}+$ $15 l(4) l(6) z^{4}+20\left(\frac{1}{2} l(4) l(5) l(6)+\frac{1}{2} l(3) l(5) l(7)\right) z^{3}+15 l(3) l(5)^{2} l(7) z^{2}$ $+6 l(3) l(4) l(5) l(6) l(7) z+l(3) l(4) l(5)^{2} l(6) l(7)$. It is easy to attach a Young diagram to a monomial $l(i) l(j) \ldots z^{k}$ as we did for $P_{6}$ but we do not understand how their coefficients are determined.

\section{Table}

We give a first few terms of the polynomials in Section 6 .

\subsection{The second Painlevé equation.}

The recurrence formula for the Yablonskii-Vorob'ev polynomials:

$$
T_{n}(t)=\frac{t T_{n-1}^{2}(t)-4\left(T_{n-1}^{\prime \prime}(t) T_{n-1}(t)-T_{n-1}^{\prime 2}(t)\right)}{T_{n-2}},
$$




$$
\begin{aligned}
T_{0}(t)= & T_{1}(t)=1 \\
T_{2}(t)= & 4+t^{3}, \\
T_{3}(t)= & -80+20 t^{3}+t^{6}, \\
T_{4}(t)= & t\left(11200+60 t^{6}+t^{9}\right), \\
T_{5}(t)= & -6272000-3136000 t^{3}+78400 t^{6}+2800 t^{9}+140 t^{12}+t^{15}, \\
T_{6}(t)= & -38635520000+19317760000 t^{3}+1448832000 t^{6}-17248000 t^{9} \\
& +627200 t^{12}+18480 t^{15}+280 t^{18}+t^{21} \\
T_{7}(t)= & -3093932441600000 t-49723914240000 t^{7}-828731904000 t^{10} \\
& +13039488000 t^{13}+62092800 t^{16}+5174400 t^{19} \\
& +75600 t^{22}+504 t^{25}+t^{28}, \\
T_{8}(t)= & -991048439693312000000-74328632976998400000 t^{3} \\
& +37164316488499200000 t^{6}+1769729356595200000 t^{9} \\
& +126696533483520000 t^{12}+407736096768000 t^{15} \\
& -6629855232000 t^{18}+124309785600 t^{21}+2018016000 t^{24} \\
& +32771200 t^{27}+240240 t^{30}+840 t^{33}+t^{36} \\
T_{9}(t)= & 5396694815443548897280000000-404752111158261672960000000 t^{3} \\
& -404752111158266167296000000 t^{6}+9636955027577765888000000 t^{9} \\
& -55850538189165977600000 t^{12}-18430315465453731840000 t^{15} \\
& -318108851847987200000 t^{18}+483515342069760000 t^{21} \\
& +8403341506560000 t^{24}+46132742656000 t^{27}+1876485811200 t^{30} \\
& +21525504000 t^{33}+160160000 t^{36}+646800 t^{39}+1320 t^{42}+t^{45}, \\
T_{10}(t)= & -558360178500369459236883660800000000 t \\
& -17947291451797589761185546240000000 t^{7} \\
& -299121524196626496019759104000000 t^{10} \\
& +11766143871370797483294720000000 t^{13} \\
& +56029256530337130872832000000 t^{16} \\
& +9157516514955772035072000000 t^{19} \\
& +136012478911949832192000000 t^{22} \\
& +716670308138534830080000 t^{25}-2587344319342182400000 t^{28} \\
& +4351638078627840000 t^{31}+182934280488960000 t^{34} \\
& +2227039406592000 t^{37}+24080581324800 t^{40}+155387232000 t^{43} \\
& +639038400 t^{46}+1544400 t^{49}+1980 t^{52}+t^{55} \\
& \\
&
\end{aligned}
$$


8.2. The fourth equation. The recurrence formula of the Okamoto polynomials:

$$
\begin{aligned}
S_{n}(x)= & \left(S_{n-1}^{\prime \prime}(x) S_{n-1}(x)-S_{n-1}^{\prime}(x)^{2}+\left(x^{2}+2 n-3\right) S_{n}(x)^{2}\right) / S_{n-2}(x), \\
S_{0}(x)= & S_{1}(x)=1, \\
S_{2}(x)= & x \\
S_{3}(x)= & 1+x^{2}, \\
S_{4}(x)= & +5 x^{2}+5 x^{4}+x^{6}, \\
S_{5}(x)= & 175+350 x^{2}+175 x^{4}+140 x^{6}+65 x^{8}+14 x^{10}+x^{12}, \\
S_{6}(x)= & 67375+134750 x^{2}+202125 x^{4}+107800 x^{6}+42350 x^{8}+20020 x^{10} \\
& +8050 x^{12}+2200 x^{14}+355 x^{16}+30 x^{18}+x^{20}, \\
S_{7}(x)= & 337211875+1011635625 x^{2}+1011635625 x^{4}+1146520375 x^{6} \\
& +838212375 x^{8}+371896525 x^{10}+118243125 x^{12}+36295875 x^{14} \\
& +11836825 x^{16}+3570875 x^{18}+854315 x^{20}+147525 x^{22} \\
& +17325 x^{24}+1295 x^{26}+55 x^{28}+x^{30}, \\
S_{8}(x)= & 28691672384375+86075017153125 x^{2}+172150034306250 x^{4} \\
& +149196696398750 x^{6}+100830734379375 x^{8}+69023966136125 x^{10} \\
& +40183246103000 x^{12}+17590898325000 x^{14}+5725722752750 x^{16} \\
& +1496006762250 x^{18}+356797140700 x^{20}+86586199700 x^{22} \\
& +21345073750 x^{24}+4874150050 x^{26}+939138200 x^{28}+143826760 x^{30} \\
& +16851835 x^{32}+1461425 x^{34}+90090 x^{36}+3710 x^{38}+91 x^{40}+x^{42},
\end{aligned}
$$$$
S_{9}(x)=46383387951666390625+185533551806665562500 x^{2}
$$$$
+278300327709998343750 x^{4}+408173813974664237500 x^{6}
$$$$
+404198095007378546875 x^{8}+265047931152379375000 x^{10}
$$$$
+134451586893661537500 x^{12}+63494998891449125000 x^{14}
$$$$
+29435148214202355625 x^{16}+12470663251834287500 x^{18}
$$$$
+4461474719087636250 x^{20}+1306669436585012500 x^{22}
$$$$
+317280487275626875 x^{24}+66794754468442000 x^{26}
$$$$
+13050553160645000 x^{28}+2532929501102000 x^{30}
$$$$
+500062887198875 x^{32}+96562055089500 x^{34}+17076096487450 x^{36}
$$$$
+2618834718500 x^{38}+336055329225 x^{40}+35218183000 x^{42}
$$$$
+2954107100 x^{44}+194128200 x^{46}+9725275 x^{48}
$$$$
+357140 x^{50}+9030 x^{52}+140 x^{54}+x^{56} \text {. }
$$ 
8.3. The third equation. The recurrence formula:

$$
\begin{aligned}
& S_{n}(a, u)= \frac{-4 u^{3}\left(\frac{\partial S_{n-1}}{\partial u}+u \frac{\partial^{2} S_{n-1}}{\partial u^{2}}\right) S_{n-1}+4 u^{4}\left(\frac{\partial S_{n-1}}{\partial u}\right)^{2}+(1+a u) S_{n-1}^{2}}{S_{n-2}}, \\
& S_{0}(a, u)= S_{1}(a, u)=1, \\
& S_{2}(a, u)=1+a u, \\
& S_{3}(a, u)=1+3 a u+3 a^{2} u^{2}+(-2+a) a(2+a) u^{3}, \\
& S_{4}(a, u)=1+6 a u+15 a^{2} u^{2}+20(-1+a) a(1+a) u^{3} \\
&+ \\
&+15(-2+a) a^{2}(2+a) u^{4}+6(-2+a) a(2+a)\left(-6+a^{2}\right) u^{5} \\
&+(-4+a)(-2+a) a^{2}(2+a)(4+a) u^{6}, \\
& S_{5}(a, u)=1+10 a u+45 a^{2} u^{2}+60 a\left(-1+2 a^{2}\right) u^{3}+210 a^{2}\left(-2+a^{2}\right) u^{4} \\
&+252(-2+a)(-1+a) a(1+a)(2+a) u^{5} \\
&+210(-2+a) a^{2}(2+a)\left(-6+a^{2}\right) u^{6} \\
&+60(-2+a) a(2+a)\left(60-27 a^{2}+2 a^{4}\right) u^{7} \\
&+45(-4+a)(-2+a) a^{2}(2+a)(4+a)\left(-8+a^{2}\right) u^{8} \\
&+ \\
&+10(-4+a)(-2+a) a^{3}(2+a)(4+a)\left(-22+a^{2}\right) u^{9} \\
&+(-6+a)(-4+a)(-2+a)^{2} a^{2}(2+a)^{2}(4+a)(6+a) u^{10}, \\
& S_{6}(a, u)=1+15 a u+105 a^{2} u^{2}+35 a\left(-4+13 a^{2}\right) u^{3}+105 a^{2}\left(-16+13 a^{2}\right) u^{4} \\
&+21 a\left(192-440 a^{2}+143 a^{4}\right) u^{5}+385(-2+a) a^{2}(2+a)\left(-28+13 a^{2}\right) u^{6} \\
&+45(-2+a) a(2+a)\left(720-968 a^{2}+143 a^{4}\right) u^{7} \\
&+45(-2+a) a^{2}(2+a)\left(5424-1892 a^{2}+143 a^{4}\right) u^{8} \\
&+35(-2+a) a(2+a)\left(-20160+18416 a^{2}-3124 a^{4}+143 a^{6}\right) u^{9} \\
&+21(-4+a)(-2+a) a^{2}(2+a)(4+a)\left(7632-2420 a^{2}+143 a^{4}\right) u^{10} \\
&+105(-4+a)(-2+a) a^{3}(2+a)(4+a)\left(2592-400 a^{2}+13 a^{4}\right) u^{11} \\
&+35(-4+a)(-2+a) a^{2}(2+a)(4+a)\left(-5760+7312 a^{2}-620 a^{4}+13 a^{6}\right) u^{12} \\
&+105(-6+a)(-4+a)(-2+a)^{2} a^{3}(2+a)^{2}(4+a)(6+a)\left(-28+a^{2}\right) u^{13} \\
&+15(-6+a)(-4+a)(-2+a)^{2} a^{2}(2+a)^{2}(4+a)(6+a)\left(240-52 a^{2}+a^{4}\right) u^{14} \\
&+(-8+a)(-6+a)(-4+a)^{2}(-2+a)^{2} a^{3}(2+a)^{2}(4+a)^{2}(6+a)(8+a) u^{15} .
\end{aligned}
$$

8.4. The fifth equation. The recurrence formula:

$$
\begin{aligned}
T_{n}(v, t) & =\frac{\left(\frac{t}{8}-v+\frac{3(n-1)}{4}\right) T_{n-1}^{2}+\frac{\partial T_{n-1}}{\partial t} T_{n-1}+t \frac{\partial T_{n-1}^{2}}{\partial t^{2}} T_{n-1}-t\left(\frac{\partial T_{n-1}}{\partial t}\right)^{2}}{T_{n-2}}, \\
T_{0} & =T_{1}=1,
\end{aligned}
$$




$$
\begin{aligned}
& 2^{3} T_{2}(v, t)=6-8 v+t, \\
& 2^{9} T_{3}(v, t)=32(1-v)(-5+4 v)(-3+4 v) \\
& +12(-5+4 v)(-3+4 v) t+24(1-v) t^{2}+t^{3}, \\
& 2^{18} T_{4}(v, t)=512(-1+v)(-3+2 v)(-7+4 v)(-5+4 v)^{2}(-3+4 v) \\
& +1536(1-v)(-3+2 v)(-7+4 v)(-5+4 v)(-3+4 v) t \\
& +240(-7+4 v)(-5+4 v)^{2}(-3+4 v) t^{2} \\
& +80(-5+4 v)\left(-45+80 v-32 v^{2}\right) t^{3} \\
& +480(-1+v)(-3+2 v) t^{4}+12(5-4 v) t^{5}+t^{6},
\end{aligned}
$$




$$
\begin{aligned}
& +92160(-2+v)(-3+2 v)(-7+4 v) \\
& \quad \times\left(-123135+333872 v-319616 v^{2}+128128 v^{3}-18304 v^{4}\right) t^{8} \\
& +17920\left(1613115-6065682 v+9304924 v^{2}\right. \\
& \left.\quad-7465920 v^{3}+3308800 v^{4}-768768 v^{5}+73216 v^{6}\right) t^{9} \\
& +1344(-7+4 v)\left(-142695+358512 v-326656 v^{2}+128128 v^{3}-18304 v^{4}\right) t^{10} \\
& +3360\left(13905-33712 v+30016 v^{2}-11648 v^{3}+1664 v^{4}\right) t^{11} \\
& +2240(-7+4 v)\left(-75+91 v-26 v^{2}\right) t^{12} \\
& +3360(-2+v)(-3+2 v) t^{13}+30(7-4 v) t^{14}+t^{15}
\end{aligned}
$$

\subsection{The sixth equation}

The recurrence formula:

$$
\begin{aligned}
& T_{n}\left(b_{1}, b_{2}, v\right)=\frac{\left\{\frac{1}{4}\left(-2 b_{1}^{2}-2 b_{2}^{2}+\left(b_{1}^{2}-b_{2}^{2}\right)\right) v+\left(n-\frac{1}{2}\right)^{2}\right\} T_{n-1}^{2}}{T_{n-2}} \\
& +\frac{\frac{1}{4}\left(v^{2}-4\right)\left\{\left(v^{2}-4\right) \frac{\partial^{2} T_{n-1}}{\partial v^{2}}+v \frac{\partial T_{n-1}}{\partial v}\right\} T_{n-1}-\left(\frac{1}{2}\left(v^{2}-4\right) \frac{\partial T_{n-1}}{\partial v}\right)^{2}}{T_{n-2}}, \\
& T_{0}\left(b_{1}, b_{2}, v\right)=T_{1}\left(b_{1}, b_{2}, v\right)=1, \\
& 4 T_{2}\left(b_{1}, b_{2}, v\right)=1-2 b_{1}^{2}-2 b_{2}^{2}+b_{1}^{2} v-b_{2}^{2} v, \\
& 64 T_{3}\left(b_{1}, b_{2}, v\right)=9-38 b_{1}^{2}+28 b_{1}^{4}-8 b_{1}^{6}-38 b_{2}^{2}+120 b_{1}^{2} b_{2}^{2}-24 b_{1}^{4} b_{2}^{2}+28 b_{2}^{4} \\
& -24 b_{1}^{2} b_{2}^{4}-8 b_{2}^{6}+15 b_{1}^{2} v-36 b_{1}^{4} v+12 b_{1}^{6} v-15 b_{2}^{2} v+12 b_{1}^{4} b_{2}^{2} v \\
& +36 b_{2}^{4} v-12 b_{1}^{2} b_{2}^{4} v-12 b_{2}^{6} v+15 b_{1}^{4} v^{2}-6 b_{1}^{6} v^{2}-30 b_{1}^{2} b_{2}^{2} v^{2} \\
& +6 b_{1}^{4} b_{2}^{2} v^{2}+15 b_{2}^{4} v^{2}+6 b_{1}^{2} b_{2}^{4} v^{2}-6 b_{2}^{6} v^{2}+b_{1}^{2} v^{3}-2 b_{1}^{4} v^{3} \\
& +b_{1}^{6} v^{3}-b_{2}^{2} v^{3}-3 b_{1}^{4} b_{2}^{2} v^{3}+2 b_{2}^{4} v^{3}+3 b_{1}^{2} b_{2}^{4} v^{3}-b_{2}^{6} v^{3}, \\
& 4096 T_{4}\left(b_{1}, b_{2}, v\right)=2025-13212 b_{1}^{2}+24364 b_{1}^{4}-14656 b_{1}^{6}+4272 b_{1}^{8}-832 b_{1}^{10} \\
& +64 b_{1}^{12}-13212 b_{2}^{2}+74648 b_{1}^{2} b_{2}^{2}-105280 b_{1}^{4} b_{2}^{2}+47552 b_{1}^{6} b_{2}^{2}-6720 b_{1}^{8} b_{2}^{2} \\
& +384 b_{1}^{10} b_{2}^{2}+24364 b_{2}^{4}-105280 b_{1}^{2} b_{2}^{4}+66080 b_{1}^{4} b_{2}^{4}-16000 b_{1}^{6} b_{2}^{4}+960 b_{1}^{8} b_{2}^{4} \\
& -14656 b_{2}^{6}+47552 b_{1}^{2} b_{2}^{6}-16000 b_{1}^{4} b_{2}^{6}+1280 b_{1}^{6} b_{2}^{6}+4272 b_{2}^{8}-6720 b_{1}^{2} b_{2}^{8} \\
& +960 b_{1}^{4} b_{2}^{8}-832 b_{2}^{10}+384 b_{1}^{2} b_{2}^{10}+64 b_{2}^{12}+5130 b_{1}^{2} v-24252 b_{1}^{4} v+23520 b_{1}^{6} v \\
& -10656 b_{1}^{8} v+2400 b_{1}^{10} v-192 b_{1}^{12} v-5130 b_{2}^{2} v+74400 b_{1}^{4} b_{2}^{2} v-51264 b_{1}^{6} b_{2}^{2} v \\
& +11040 b_{1}^{8} b_{2}^{2} v-768 b_{1}^{10} b_{2}^{2} v+24252 b_{2}^{4} v-74400 v b_{1}^{2} b_{2}^{4} v \\
& +8640 b_{1}^{6} b_{2}^{4} v-960 b_{1}^{8} b_{2}^{4} v-23520 b_{2}^{6} v+51264 b_{1}^{2} b_{2}^{6} v-8640 b_{1}^{4} b_{2}^{6} v \\
& +10656 b_{2}^{8} v-11040 b_{1}^{2} b_{2}^{8} v+960 b_{1}^{4} b_{2}^{8} v-2400 b_{2}^{10} v+768 b_{1}^{2} b_{2}^{10} v+192 b_{2}^{12} v \\
& +5175 b_{1}^{4} v^{2}-15720 b_{1}^{6} v^{2}+11160 b_{1}^{8} v^{2}-2880 b_{1}^{10} v^{2}+240 b_{1}^{12} v^{2} \\
& -10350 b_{1}^{2} b_{2}^{2} v^{2}+15720 b_{1}^{4} b_{2}^{2} v^{2}+4800 b_{1}^{6} b_{2}^{2} v^{2}-4800 b_{1}^{8} b_{2}^{2} v^{2}+480 b_{1}^{10} b_{2}^{2} v^{2}
\end{aligned}
$$




$$
\begin{aligned}
& +5175 b_{2}^{4} v^{2}+15720 b_{1}^{2} b_{2}^{4} v^{2}-31920 b_{1}^{4} b_{2}^{4} v^{2}+7680 b_{1}^{6} b_{2}^{4} v^{2}-240 b_{1}^{8} b_{2}^{4} v^{2} \\
& -15720 b_{2}^{6} v^{2}+4800 b_{1}^{2} b_{2}^{6} v^{2}+7680 b_{1}^{4} b_{2}^{6} v^{2}-960 b_{1}^{6} b_{2}^{6} v^{2}+11160 b_{2}^{8} v^{2} \\
& -4800 b_{1}^{2} b_{2}^{8} v^{2}-240 b_{1}^{4} b_{2}^{8} v^{2}-2880 b_{2}^{10} v^{2}+480 b_{1}^{2} b_{2}^{10} v^{2}+240 b_{2}^{12} v^{2} \\
& +45 b_{1}^{2} v^{3}-280 b_{1}^{4} v^{3}+6940 b_{1}^{6} v^{3}-6360 b_{1}^{8} v^{3}+1840 b_{1}^{10} v^{3}-160 b_{1}^{12} v^{3} \\
& -45 b_{2}^{2} v^{3}-18420 b_{1}^{4} b_{2}^{2} v^{3}+10800 b_{1}^{6} b_{2}^{2} v^{3}-1200 b_{1}^{8} b_{2}^{2} v^{3}+280 b_{2}^{4} v^{3} \\
& +18420 b_{1}^{2} b_{2}^{4} v^{3}-4320 b_{1}^{6} b_{2}^{4} v^{3}+480 b_{1}^{8} b_{2}^{4} v^{3}-6940 b_{2}^{6} v^{3}-10800 b_{1}^{2} b_{2}^{6} v^{3} \\
& +4320 b_{1}^{4} b_{2}^{6} v^{3}+6360 b_{2}^{8} v^{3}+1200 b_{1}^{2} b_{2}^{8} v^{3}-480 b_{1}^{4} b_{2}^{8} v^{3}-1840 b_{2}^{10} v^{3} \\
& +160 b_{2}^{12} v^{3}+975 b_{1}^{4} v^{4}-2490 b_{1}^{6} v^{4}+2115 b_{1}^{8} v^{4}-660 b_{1}^{10} v^{4}+60 b_{1}^{12} v^{4} \\
& -1950 b_{1}^{2} b_{2}^{2} v^{4}+2490 b_{1}^{4} b_{2}^{2} v^{4}-3900 b_{1}^{6} b_{2}^{2} v^{4}+1500 b_{1}^{8} b_{2}^{2} v^{4}-120 b_{1}^{10} b_{2}^{2} v^{4} \\
& +975 b_{2}^{4} v^{4}+2490 b_{1}^{2} b_{2}^{4} v^{4}+3570 b_{1}^{4} b_{2}^{4} v^{4}-840 b_{1}^{6} b_{2}^{4} v^{4}-60 b_{1}^{8} b_{2}^{4} v^{4} \\
& -2490 b_{2}^{6} v^{4}-3900 b_{1}^{2} b_{2}^{6} v^{4}-840 b_{1}^{4} b_{2}^{6} v^{4}+240 b_{1}^{6} b_{2}^{6} v^{4}+2115 b_{2}^{8} v^{4} \\
& +1500 b_{1}^{2} b_{2}^{8} v^{4}-60 b_{1}^{4} b_{2}^{8} v^{4}-660 b_{2}^{10} v^{4}-120 b_{1}^{2} b_{2}^{10} v^{4}+60 b_{2}^{12} v^{4}+81 b_{1}^{2} v^{5} \\
& -342 b_{1}^{4} v^{5}+543 b_{1}^{6} v^{5}-396 b_{1}^{8} v^{5}+126 b_{1}^{10} v^{5}-12 b_{1}^{12} v^{5}-81 b_{2}^{2} v^{5} \\
& -45 b_{1}^{4} b_{2}^{2} v^{5}+504 b_{1}^{6} b_{2}^{2} v^{5}-390 b_{1}^{8} b_{2}^{2} v^{5}+48 b_{1}^{10} b_{2}^{2} v^{5}+342 b_{2}^{4} v^{5}+45 b_{1}^{2} b_{2}^{4} v^{5} \\
& +540 b_{1}^{6} b_{2}^{4} v^{5}-60 b_{1}^{8} b_{2}^{4} v^{5}-543 b_{2}^{6} v^{5}-504 b_{1}^{2} b_{2}^{6} v^{5}-540 b_{1}^{4} b_{2}^{6} v^{5}+396 b_{2}^{8} v^{5} \\
& +390 b_{1}^{2} b_{2}^{8} v^{5}+60 b_{1}^{4} b_{2}^{8} v^{5}-126 b_{2}^{10} v^{5}-48 b_{1}^{2} b_{2}^{10} v^{5}+12 b_{2}^{12} v^{5}+16 b_{1}^{4} v^{6} \\
& -40 b_{1}^{6} v^{6}+33 b_{1}^{8} v^{6}-10 b_{1}^{10} v^{6}+b_{1}^{12} v^{6}-32 b_{1}^{2} b_{2}^{2} v^{6}+40 b_{1}^{4} b_{2}^{2} v^{6}-68 b_{1}^{6} b_{2}^{2} v^{6} \\
& +30 b_{1}^{8} b_{2}^{2} v^{6}-6 b_{1}^{10} b_{2}^{2} v^{6}+16 b_{2}^{4} v^{6}+40 b_{1}^{2} b_{2}^{4} v^{6}+70 b_{1}^{4} b_{2}^{4} v^{6}-20 b_{1}^{6} b_{2}^{4} v^{6} \\
& +15 b_{1}^{8} b_{2}^{4} v^{6}-40 b_{2}^{6} v^{6}-68 b_{1}^{2} b_{2}^{6} v^{6}-20 b_{1}^{4} b_{2}^{6} v^{6}-20 b_{1}^{6} b_{2}^{6} v^{6}+33 b_{2}^{8} v^{6} \\
& +30 b_{1}^{2} b_{2}^{8} v^{6}+15 b_{1}^{4} b_{2}^{8} v^{6}-10 b_{2}^{10} v^{6}-6 b_{1}^{2} b_{2}^{10} v^{6}+b_{2}^{12} v^{6},
\end{aligned}
$$

Remark. - $[2,8]$ was published as [1]

\section{Bibliography}

[1] M. Noumi, S. Okada, K. Окамото \& H. Umemura, "Special polynomials associated with the Painlevé equations. II", in Integrable systems and algebraic geometry (Kobe/Kyoto, 1997), World Scientific, 1998, p. 349-372.

[2] M. Noumi \& K. Окамото, "On the Umemura polynomials", in preparation.

[3] K. Окамото, "Studies on the Painlevé equations III", Math. Ann. 275 (1986), p. 221255.

[4] - "Studies on the Painlevé equations I", Ann. Mat. Pura Appl. 146 (1987), p. $337-381$.

[5] — "Studies on the Painlevé equations II", Jap. J. Math. 13 (1987), p. 47-76.

[6] _ "Studies on the Painlevé equations IV", Funk. Ekv. 30 (1987), p. 305-332.

[7] H. Umemura, "Irreducibility of the Painlevé equations - Evolution in the past 100 years", "in this volume". published as "100 years of the Painlevé equation", Sûgaku 51 (1999), no. 4, 395-420.

[8] - "Special polynomials associated with the Painlevé equations II", in preparation.

[9] _ , "On the irreducibility of the first differential equation of Painlevé", in Algebraic geometry and Commutative algebra in honor of Masayoshi Nagata, Konokuniya Company Ltd, 1988, p. 101-119. 
Special polynomials associated with the Painlevé equations I

[10] H. Umemura \& H. Watanabe, "Solutions of the second and fourth Painlevé equations I", Nagoya Math. J. 148 (1997), p. 151-198.

[11] A. P. Vorob'Ev, "On rational solutions of the second Painlevé equation", Differ. Uravn 1 (1965), p. 58-59.

[12] E. T. Whittaker \& G. N. Watson, A course of modern analysis, Cambridge University Press, 1935.

[13] A. I. Yablonskit, "On rational solutions of the second Painlevé equation", Vesti A.N. BSSR., Ser. Fiz-Tekh. Nauk. 3 (1959), p. 30-35, in Russian. 